\title{
Virus-mediated delivery of antibody targeting TAR DNA-binding protein-43 mitigates associated neuropathology
}

\author{
Silvia Pozzi, ${ }^{1}$ Sai Sampath Thammisetty, ${ }^{1}$ Philippe Codron, ${ }^{1,2}$ Reza Rahimian, ${ }^{1}$ Karine Valérie Plourde, ${ }^{1}$ Geneviève Soucy, ${ }^{1}$ \\ Christine Bareil, ${ }^{1}$ Daniel Phaneuf, ${ }^{1}$ Jasna Kriz, ${ }^{1,3}$ Claude Gravel, ${ }^{1,3}$ and Jean-Pierre Julien ${ }^{1,3}$
}

${ }^{1}$ CERVO Brain Research Centre, Québec, Québec, Canada. ${ }^{2}$ MITOVASC Institute, Centre National de la Recherche Scientifique (CNRS) 6015, INSERM U1083, University of Angers, Angers, France. ${ }^{3}$ Department of Psychiatry and Neuroscience, Université Laval, Québec City, Québec, Canada.

\begin{abstract}
The cytoplasmic aggregation of TAR DNA-binding protein-43 (TDP-43) is a hallmark of degenerating neurons in amyotrophic lateral sclerosis (ALS) and subsets of frontotemporal dementia (FTD). In order to reduce TDP-43 pathology, we generated single-chain (scFv) antibodies against the RNA recognition motif 1 (RRM1) of TDP-43, which is involved in abnormal protein self-aggregation and interaction with p65 NF-кB. Virus-mediated delivery into the nervous system of a scFv antibody, named VH7Vk9, reduced microgliosis in a mouse model of acute neuroinflammation and mitigated cognitive impairment, motor defects, TDP-43 proteinopathy, and neuroinflammation in transgenic mice expressing ALS-linked TDP-43 mutations. These results suggest that antibodies targeting the RRM1 domain of TDP-43 might provide new therapeutic avenues for the treatment of ALS and FTD.
\end{abstract}

\section{Introduction}

Amyotrophic lateral sclerosis (ALS) is a fatal neurodegenerative disease characterized by the selective degeneration of both upper and lower motor neurons. Middle-aged patients (average age of 55 years) present to the clinician with main muscle-related symptomatology. The disease then progresses to muscle atrophy followed by complete paralysis, and death generally occurs by respiratory failure 3 to 5 years after symptom onset. Diagnosis, which is still based on clinical evaluations, aims to exclude other disorders that resemble ALS and is thus often delayed by about 1 year from the first appearance of symptoms $(1,2)$. Ninety percent of patients with ALS have sporadic-origin ALS (sALS), while ten percent have familial inherited mutations (fALS) $(1,3)$.

TAR DNA-binding protein 43 (TDP-43) is a DNA/RNA-binding protein that is highly and ubiquitously expressed, with main localization in the cell nucleus (4). Thanks to its 2 RNA recognition domains (RRM1 and RRM2), the protein is a multifunctional factor involved in different aspects of RNA metabolism such as transcription, splicing, stabilization, and transport (4). Although mutations in TDP-43 are very rare, occurring in $3 \%$ of patients with fALS and $1.5 \%$ of those with sALS, more than $90 \%$ of ALS cases (fALS and sALS) show a pathological behavior of this protein called TDP-43 proteinopathy $(4,5)$. This event was first described in 2006 (6) as a consistent mis-

Conflict of interest: JPJ, CG, and SP are owners of US patent application no. 15/532,909 titled "TDP-43-binding polypeptides useful for the treatment of neurodegenerative diseases." JPJ is chief scientific officer of ImStar Therapeutics. Copyright: @ 2019 American Society for Clinical Investigation Submitted: July 31, 2018; Accepted: January 15, 2019.

Reference information: J Clin Invest. 2019;129(4):1581-1595. https://doi.org/10.1172/JCI123931. localization and aggregation of the protein in the cytoplasm where TDP-43 can form hyperphosphorylated, fragmented, and ubiquitinated inclusions that impair the physiological function of the protein (6-8). TDP-43 proteinopathy is not exclusive to ALS disease. It is in fact present in $50 \%$ of patients with frontotemporal lobar dementia (FTLD) $(5,9)$. FTLD, also referred to as frontotemporal dementia (FTD), is a midlife-onset disease that is clinically heterogeneous and characterized by changes in behavior, personality, and/or language (9). Because of TDP- 43 proteinopathy, ALS and FTD are now considered a disease continuum, with $50 \%$ of ALS patients presenting with cognitive impairment (15\%-20\% recognized as FTD) and $15 \%$ of FTD patients having motor impairments $(3,5)$. Interestingly, TDP-43 proteinopathy has also been observed in other neurodegenerative disorders such as Alzheimer's disease (AD), Parkinson's disease (PD), and Huntington's disease (HD) $(4,10)$.

Different studies have highlighted the sensitivity of the RRM1 domain in inducing TDP-43 proteinopathy. Oxidation or misfolding of this domain results in cytosolic mislocalization with irreversible protein aggregation $(11,12)$. Apart from RNA metabolism, the RRM1 domain is also responsible for the interaction between TDP-43 and the p65 subunit of NF-KB (13). This interaction mediates overactivation of the NF- $\kappa$ B pathway, which induces an increased vulnerability of neurons to toxic injuries and a hyperactive inflammatory response of glial cells (13). Both TDP-43 and p65 proteins are overexpressed in patients with sALS $(13,14)$, and the downregulation of TDP- 43 can reduce NF- $\mathrm{kB}$ activation (13).

Here, we describe the generation of single-chain ( $\mathrm{scFv}$ ) antibodies specifically against the RRM1 domain of TDP- 43 , with the dual aim to (a) block TDP-43-p65 interaction and thereby reduce NF- $\kappa B$ activation, and (b) interfere with protein aggregation. Single-chain antibodies are small molecules made by the linking of 
A

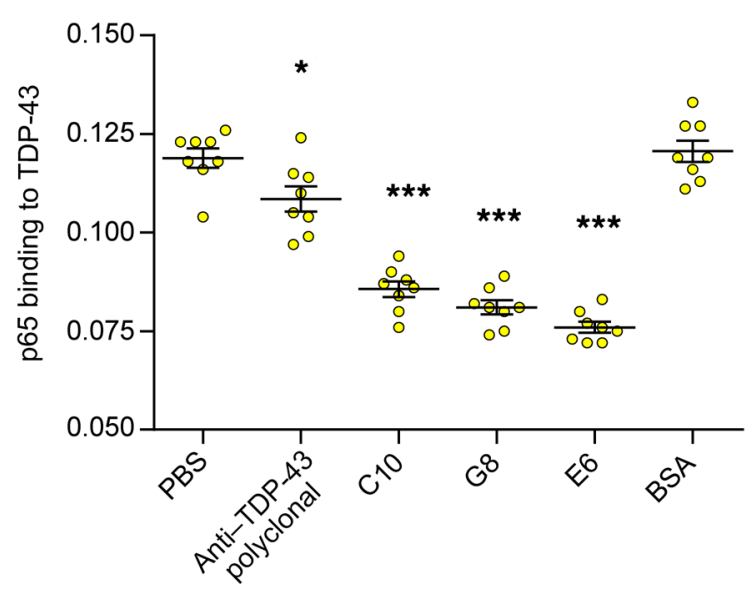

B

pscFv9 plasmid

\begin{tabular}{|c|c|c|c|}
\hline CMV promoter & $\begin{array}{c}\text { lgH } \\
\text { domain }\end{array}$ & VH----linker---Vk & $\begin{array}{c}\text { Myc } \\
\text { tag }\end{array}$ \\
\hline
\end{tabular}

E
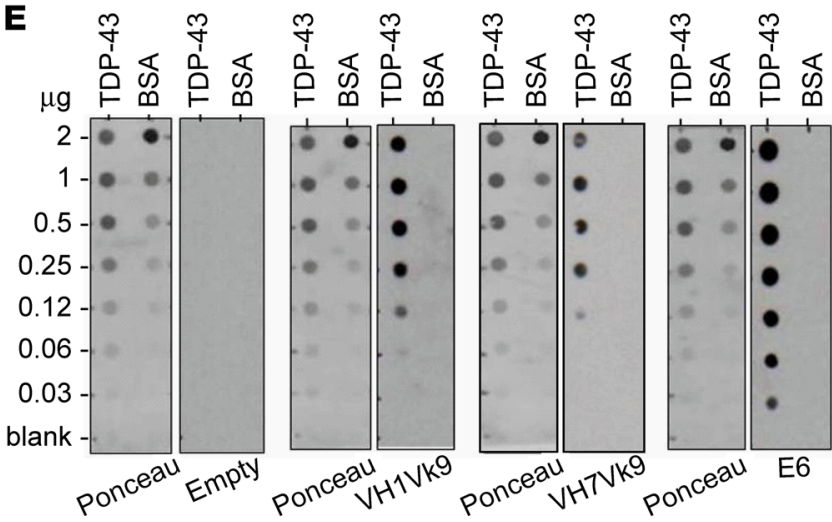

C

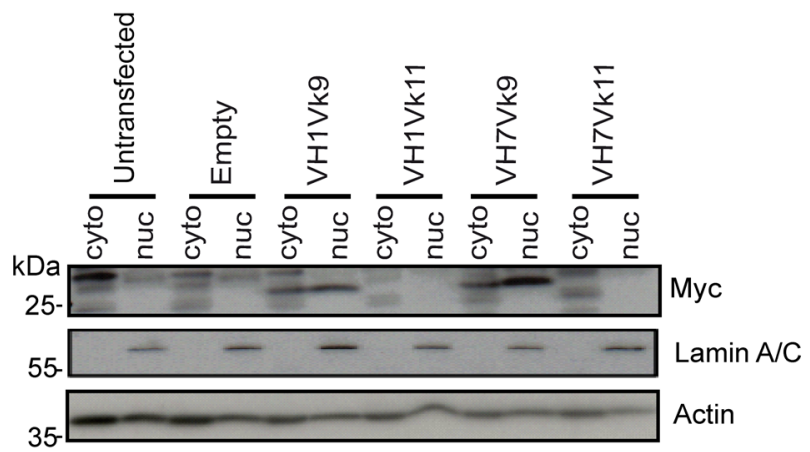

D

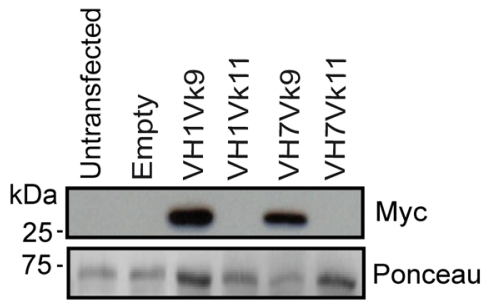

$\mathbf{F}$

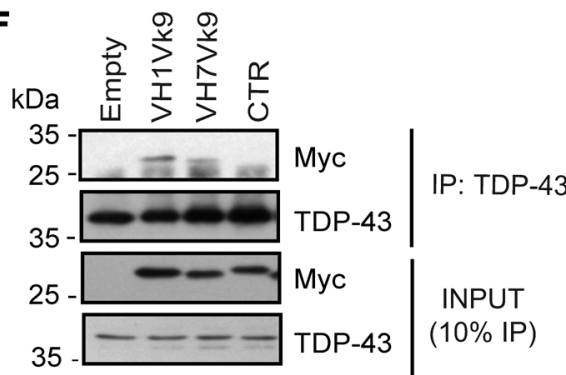

Figure 1. E6-derived scFv antibodies are able to recognize TDP-43. (A) Binding of p65 to TDP-43 was measured in the presence of PBS or anti-TDP-43 $\mathrm{N}$-terminal antibody (Proteintech), monoclonal antibodies against RRM1 TDP-43 (named C10, G8, and E6), or BSA. $n=8$ wells per condition (dots). Oneway ANOVA $P<0.0001 ;{ }^{*} P<0.05$ and ${ }^{* * *} P<0.001$ versus PBS, by Tukey's multiple comparisons test. Data represent the mean \pm SEM. (B) Schematic representation of the pscFv9 plasmid used for scFv production and expression. (C) Representative Western blot of cytoplasmic and nuclear fractions of Hek293 cells. Anti-Myc antibody revealed scFv and laminin A/C or actin in the different fractions. (D) Representative image of media from transfected Hek293 cells probed with anti-Myc antibody. Ponceau staining was used as a reference. (E) Different concentrations of TDP-43 (1-206 aa, Proteintech) or BSA were loaded onto a dot blot membrane. Immunoblots were performed with media containing pscFv9-transfected Hek293 cells and E6 monoclonal antibody. Signals were revealed with anti-Myc-HRP antibody for scFv conditions or anti-mouse HRP for E6. Ponceau staining was used as a reference. (F) Representative blot of TDP-43 immunoprecipitation in pscFv9-transfected Hek293 cells. Experiments in C, D, and F were conducted more than 3 times. Empty, no scFv; CTR, control D1.3 scFv.

variable heavy and light chains of the antigen-binding domain of an antibody (15). Given their small size, good tissue penetration, and low immunogenicity (16), scFv antibodies have been produced for different neurodegenerative disorders such as AD (17-20), Lewy body disease, PD $(21,22), \operatorname{HD}(23,24)$, and ALS (25-27). We report for the first time to our knowledge the in vivo therapeutic effects of a scFv antibody against TDP-43 in transgenic mouse models of ALS/FTD.

\section{Results}

Selection of monoclonal antibodies against the RRM1 domain of $T D P-43$. We generated monoclonal antibodies against the RRM1 domain of TDP-43, because this domain contributes to protein aggregation $(11,12)$ and to the interaction with p65 NF- $\mathrm{BB}(13)$. Among 8 hybridoma clones produced, only 3 of them, named C10, G8, and E6, were able to clearly detect TDP-43 on immunoblots after SDS-PAGE (Supplemental Figure 1A; supplemental material available online with this article; https://doi.org/10.1172/ JCI123931DS1). We then examined the ability of these 3 antibodies to disrupt the interaction between TDP-43 and p65 NF-кB. We performed an ELISA with human recombinant TDP-43 and human recombinant p65 protein (Supplemental Figure 1B). The results revealed that the 2 proteins have a direct interaction that is TDP-43 dose dependent. Using the same ELISA system, we incubated p65 recombinant protein together with a fixed amount of monoclonal antibodies against the RRM1 domain of TDP-43 (Fig- 
A

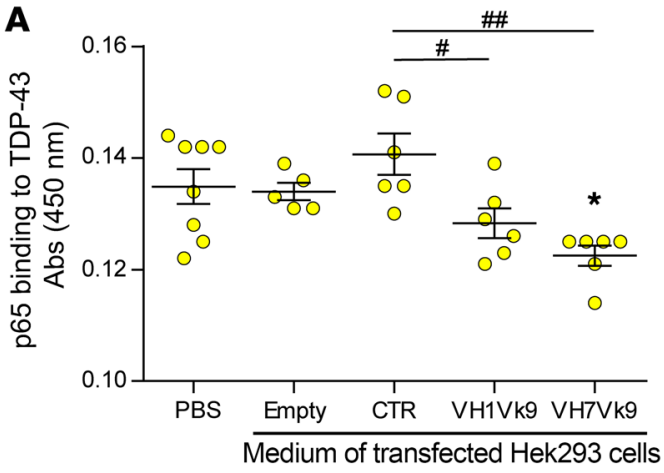

C

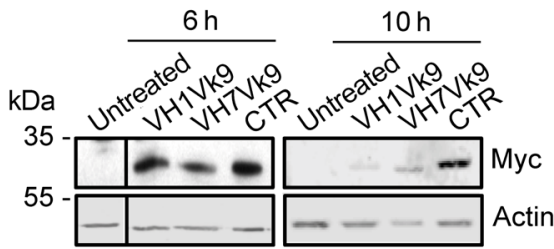

B

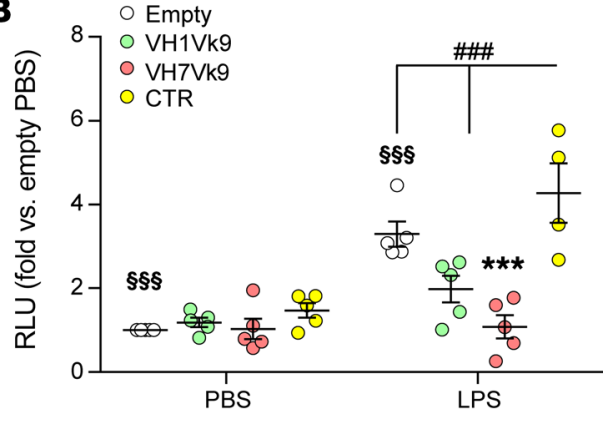

D $\quad{ }^{120} 7^{\circ} \mathrm{VH} 1 \mathrm{Vk} 9$

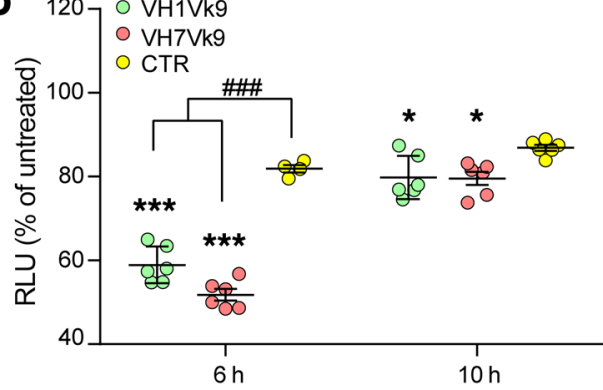

Figure 2. ScFv antibodies disrupt the interaction between TDP-43 and p65 and reduce NF-kB activity. (A) Binding of p65 to TDP-43 was measured in the presence of equal volumes of PBS or media from pscFv9-transfected Hek293cells. $n=5-8$ wells per condition (dots). One-way ANOVA $P=0.002 ;{ }^{*} P<0.05$ versus PBS; ${ }^{\#} P<0.05$ or ${ }^{\# \# P}<0.001$ versus control scFv by Tukey's multiple comparisons test. (B) BV2 cells were transfected with pscFv9, stimulated with LPS or PBS, and lysated for the luciferase assay. $n=4-5$ individual experiments (dots). Two-way ANOVA $P=0.0002 ; \$ \$ \$<0.001$ versus LPS; ${ }^{* * *} P<0.001$ versus empty; and ${ }^{\# \# P}<0.001$ versus control scFv, by Tukey's multiple comparisons test. (C and D) BV2 p65-luc cells were treated or not for 6 hours or 10 hours with scFv antibodies, together with 4 hours of LPS treatment. (C) Representative Western blots of total lysates of Myc-tag scFv inside the cells and actin ( $n=3$ pooled experiments). The blot of the untreated cells at 6 hours was run on the same gel but was noncontiguous. (D) NF- $\kappa B$ activity assessed by luciferase assay. $n=4-6$ replicates (dots) from 2 independent experiments ( $2-3$ wells each). Two-way ANOVA $P<0.0001 ;{ }^{*} P<0.05$ and ${ }^{* * *} P<0.001$ versus untreated cells; \#\# $P<0.001$ versus control scFv, by Tukey's multiple comparisons test. Data represent the mean $\pm S E M$. RLU, relative luminescence units; CTR, control D1.3 scFv.

ure 1A). All 3 of the selected monoclonal antibodies against RRM1 were able to disrupt TDP-43/p65 interaction more efficiently than the polyclonal antibody against the TDP- $43 \mathrm{~N}$-terminal. Among our monoclonal antibodies, the $\mathrm{E} 6$ clone was the most effective at interfering with the binding. Therefore, we derived scFv antibodies from this monoclonal antibody.

Production and characterization of E6-derived single-chain antibodies. Reverse transcription PCR (RT-PCR) of cDNA from E6 hybridoma cells using degenerative primers led to production of 2 different heavy chains, named VH1 and VH7, and 2 different light chains, named Vk9 and Vk11. Four different plasmids were therefore produced encoding all different combinations of heavy and light chains purified from the E6 clone. Figure 1B shows a schematic representation of the plasmid (pscFv9) (26) used for the production of scFv antibodies. The plasmid contains a CMV promoter, an IgH domain for proper folding and secretion, a singlechain antibody made of variable heavy and light chains, and a human c-Myc tag for immunodetection. As shown in Figure 1C, two plasmids encoding for VH1Vk9 and VH7Vk9 fusion proteins were able to yield the expression of scFv antibodies in transfected human embryonic kidney 293 (Hek293) cells. The scFv antibody was detected in both nuclear and cytoplasmic fractions. The recombinant scFv antibodies were also secreted into the medium (Figure 1D). From the same clone, we therefore produced 2 dif- ferent ScFv antibodies against the TDP-43 RRM1 domain. Both antibodies shared the same variable light chain VK9 but had 1-aa difference in the heavy chain, with VH1 owning a glutamic acid (E) after the cloning site and VH7 having a glutamine $(\mathrm{Q})$ in the same position (Supplemental Figure 1C) (GenBank accession numbers MK210239 and MK210240).

To test the ability of the scFv antibodies to bind TDP-43, we used the medium of vector-transfected cells as a source of $\mathrm{scFv}$ antibodies. Different concentrations of TDP-43 peptide (1-206 aa containing RRM1) or BSA were applied on a dot blot membrane and probed with the medium of transfected cells. Figure 1E shows that the media containing VH1Vk9 or VH7Vk9 were able to detect TDP-43 specifically, whereas no cross-reaction was observed on spots loaded with BSA, confirming the specificity of the scFv antibody in recognizing only TDP-43. Full-length E6 antibody was used as a positive control, confirming the ability of the monoclonal antibody to recognize TDP- 43 . The specific interaction between $\mathrm{scFv}$ and TDP- 43 was also confirmed by immunoprecipitation of TDP-43 from Hek293-transfected cells expressing scFv antibodies (Figure 1F). A clear band for the $\mathrm{scFv}$ (c-Myc-tagged) at $28 \mathrm{kDa}$ was indeed detectable when cells were transfected with E6-derived scFv VH1Vk9 and VH7Vk9. This band was absent in cells transfected with the empty plasmid or in cells transfected with a control scFv (D1.3) encoding for a scFv antibody against 
A

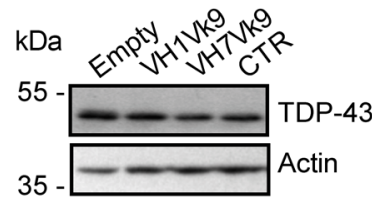

B

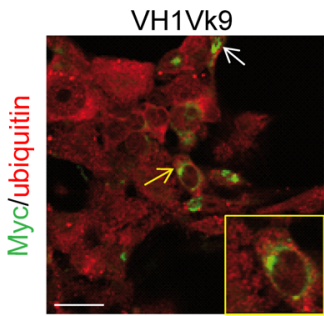

C

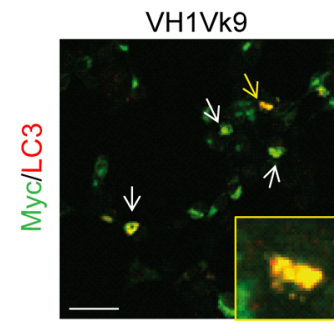

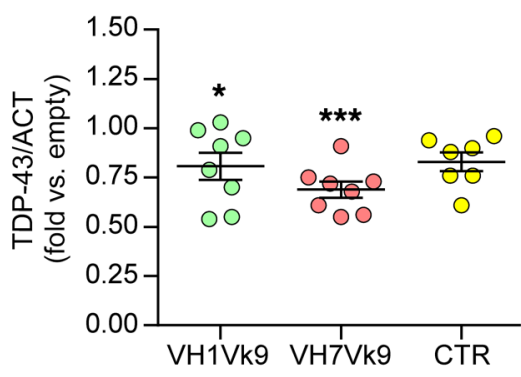

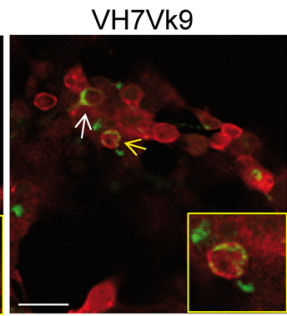

VH7Vk9

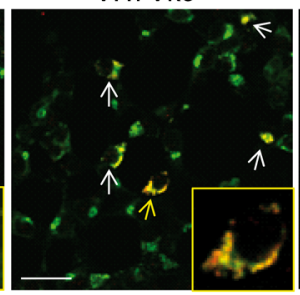

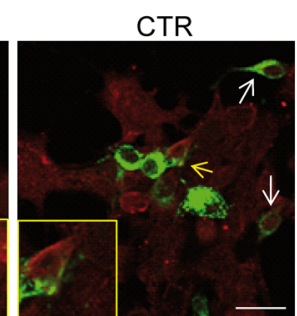

CTR

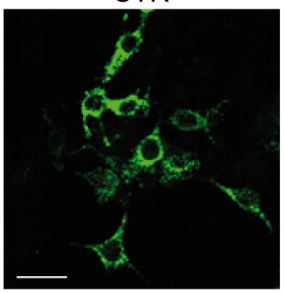

D
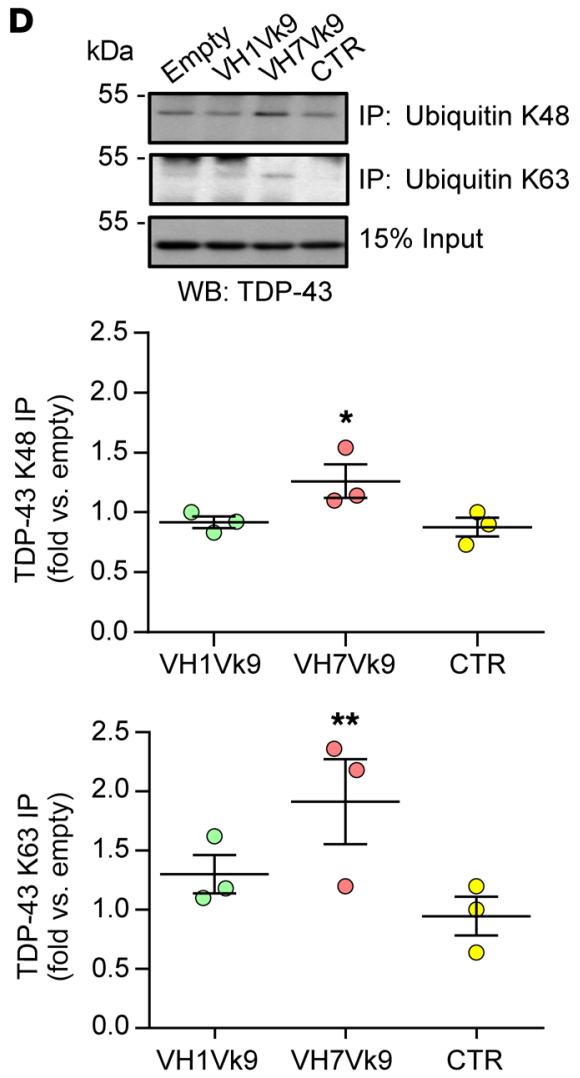

Figure 3. ScFv antibodies mediate TDP-43 degradation. (A) Representative blot and quantification of TDP-43 levels in Hek293 cells transfected with pscFv9 plasmid. Graph shows quantification of TDP-43 normalized to actin and expressed as the fold change versus empty vector. $n=7-9$ independent experiments (dots). One-way ANOVA $P=0.0003$; ${ }^{*} P<0.05$ and ${ }^{*}{ }^{*} P<0.0001$ versus empty transfected, by Tukey's multiple comparisons test. (B and C) Representative merged images of colocalization between scFv (Myc, green) and ubiquitin or LC3 (red). Arrows show cells with colocalization; yellow arrows indicate cells enlarged 2.5 times in the insets. Scale bars: $20 \mu \mathrm{m}$. The experiment was performed twice. (D) Representative blots and quantification of immunoprecipitation for all K48-polyubiquitinated (proteasome) or K63-polyubiquitinated (autophagy) proteins and Western blot for TDP-43. Graphs indicate the quantification of TDP-43 signal in immunoprecipitation experiments, expressed as the fold change versus empty vector. $n=3$ independent experiment (dots). K63-ubiquitinated TDP-43: $P=0.0082$, by 1 -way ANOVA; ${ }^{*} P<0.01$ versus empty vector-transfected cells, by Dunnett's multiple comparisons test. K48-ubiquitinated TDP-43: $P=0.0128$, by 1 -way ANOVA; ${ }^{*} P<0.05$ versus empty vector-transfected cells, by Dunnett's multiple comparisons test. Data represent the mean \pm SEM. Empty, no scFv (A and D); CTR, D1.3 scFv (B and C) or 8 H11 anti-GFP scFv (A and D).

chicken lysozyme. Immunoprecipitation of TDP-43 from nuclear and cytoplasmic fractions of transfected cells revealed that the binding of VH1Vk9 or VH7Vk9 to TDP-43 occurred predominantly in the cytoplasm (Supplemental Figure 1D).

Since the $\mathrm{scFv}$ antibodies were generated against the RRM1 domain of TDP-43, we examined whether the binding of $\mathrm{scFv}$ antibodies altered the ability of TDP- 43 to bind RNAs. Thus, we performed UV cross-linking immunoprecipitation (UV-CLIP) experiments in pscFv9-transfected Hek293 cells and verified the ability of TDP- 43 protein to bind its own RNA (28) in the presence of the scFv antibodies. As shown in Supplemental Figure 1E, only the presence of VH1Vk9 antibody was able to reduce the binding between the protein and the RNA, whereas no alterations were observed in the presence of VH7Vk9 antibody.

Single-chain antibodies reduced $p 65 \mathrm{NF}-\kappa \mathrm{B}$ activation. To determine whether E6-derived $\mathrm{scFv}$ antibodies against TDP-43 were able to disrupt the interaction between p 65 NF- $\mathrm{BB}$ and TDP-43, we performed an ELISA, in which human recombinant p65 was incubated with media of transfected cells containing scFv antibodies (Supplemental Figure 2A). Figure 2A shows that both
VH1Vk9 and VH7Vk9 were able to disrupt the protein-protein interaction between TDP-43 and p65. We then examined the effect on NF- $\kappa$ B activity of $\mathrm{scFv}$ antibodies against TDP- 43 in BV2 cells harboring a NF- $\kappa \mathrm{B}-$ luciferase reporter gene (29). BV2 cells were transfected with $\mathrm{scFv}$ vectors or controls and stimulated for 4 hours with LPS or PBS (Figure 2B). We observed that NF- $\mathrm{BB}$ activity was strongly increased after LPS treatment in empty and control scFv vector-transfected cells, whereas activation was reduced in the presence of VH1Vk9 and VH7Vk9 antibodies. It is well established that microglia-induced toxicity toward neurons is strongly dependent on microglial NF- $\kappa \mathrm{B}$ activation $(13,30)$. Therefore, we tested the effect of these scFv antibodies in such a paradigm. As shown in Supplemental Figure 2B, expression of VH7Vk9 scFv in LPS-treated BV2 cells rescued the toxicity of microglial medium toward N2A neuronal cells.

Since the scFv antibodies can be secreted from transfected cells, we examined whether VH1Vk9 or VH7Vk9 scFv antibodies were able to penetrate cells and inhibit NF- $\kappa \mathrm{B}$ activity. We first purified these scFv antibodies from media of Hek293-transfected cells. The preparation yielded a single $28-\mathrm{kD}$ a protein detectable by Coomassie 
A

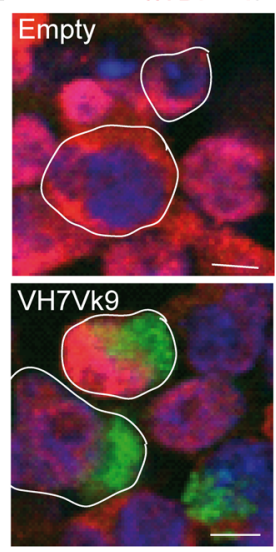

hTDP-43/Myc/Hoechst

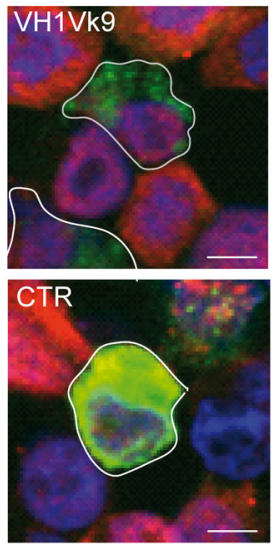

B

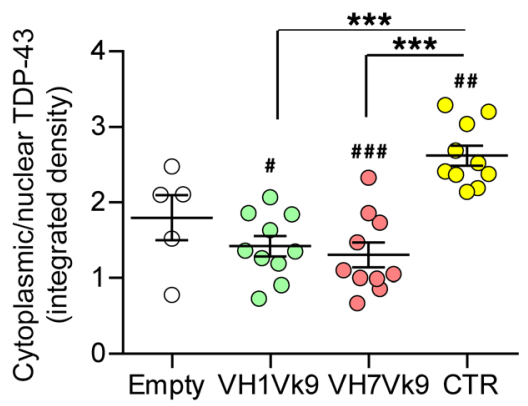

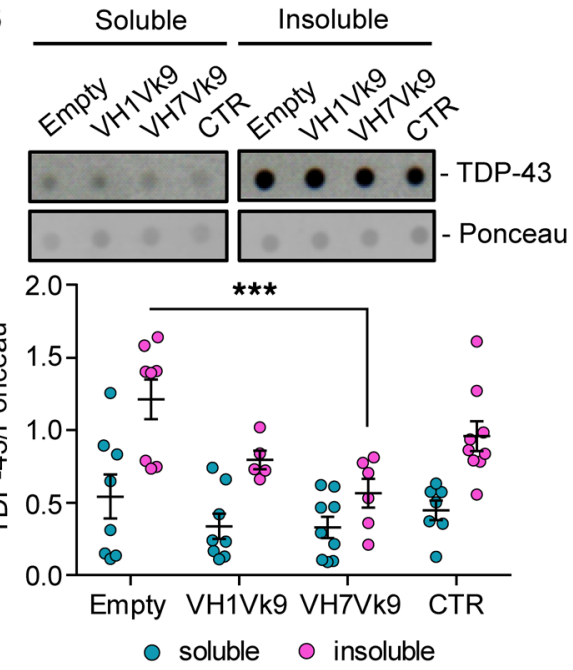

Figure 4. ScFv antibodies reduce TDP-43 mislocalization and aggregation. (A) Representative enlarged images and TDP-43 mislocalization determined by quantification of the integrated density of TDP-43 immunoreactivity in nuclear and cytoplasmic compartments. Images show merged scFv (Myc, green), hTDP-43 (red), and Hoechst (nuclei, blue) channels, and lines circumscribe the perimeter of the representative cells. Scale bars: $5 \mu \mathrm{m}$. Data in the graph represent the cytoplasmic to nuclear ratio quantified in at least 200 cells per frame. $n=5-10$ different frames (dots) analyzed from 2 independent experiments. One-way ANOVA $P<0.0001$; ${ }^{* *} P<0.001$ versus control-transfected cells; ${ }^{\#} P<0.05$ and ${ }^{\# \#} P<0.001$ versus empty vector-transfected cells, by Tukey's multiple comparisons test. (B) Representative image and quantification of dot blot analysis for soluble or insoluble TDP-43 in transfected Hek293 cells treated with $50 \mu$ M EA. Data represent TDP-43 immunoreactivity normalized to Ponceau from 5 to 9 replicates (dots) from 3 independent experiments. Two-way ANOVA interaction $P=0.249$, scFv $P=0.0014$, and soluble/insoluble $P<0.0001$; ${ }^{* * *} P<0.001$ versus empty vector-transfected cells, by Tukey’s multiple comparisons test. Data represent the mean \pm SEM. Empty, no scFv, CTR, control D1.3 scFv.

blue after SDS-PAGE and anti-Myc antibody (Supplemental Figure 2, $\mathrm{C}$ and D). The purified scFv antibodies were added to the medium $(5 \mu \mathrm{g} / \mathrm{ml})$ of BV2 p65-luciferase cells for 6 or 10 hours, together with 4 hours of LPS treatment, and NF- $\mathrm{BB}$ activity was then measured (Supplemental Figure 2E). In extracts of cells treated with scFv antibodies, we were able to detect a band corresponding to the intracellular scFv antibody at 6 hours and, to a lesser extent, after 10 hours of treatment (Figure 2C). The levels of intracellular VH1Vk9 or VH7Vk9 scFv antibodies were associated with a reduction of $\mathrm{NF}-\kappa \mathrm{B}$ activity (Figure 2D). These results indicate that $\mathrm{scFv}$ antibodies are able to penetrate cells and exert functional effects.

Single-chain antibodies drove TDP-43 to proteasome- and autophagy-mediated degradation. Transfection of E6-derived vectors encoding $\mathrm{ScFV}$ antibodies in Hek293 cells led to a decrease in total cellular levels of TDP-43 compared with empty vectortransfected cells (Figure 3A). This reduction was not sufficient to affect cell viability (Supplemental Figure 3A). We then questioned the mechanism underlying the reduction of TDP-43 levels mediated by single-chain antibodies. First, we considered the possibility that secreted scFv could facilitate the release of TDP43 into the medium. As expected, we found that $\mathrm{scFv}$ antibodies localized mainly in the cytoplasm with the trans-Golgi network where proteins are processed for secretion (Supplemental Figure $3 \mathrm{~B})$. However, our results revealed no changes in the levels of TDP-43 in the media due to expression of VH1Vk9 or VH7Vk9 scFv antibodies (data not shown). As an alternative mechanism, we investigated whether intracellular $\mathrm{scFv}$ antibodies, binding to TDP-43, might promote its degradation via proteasomal or autophagic pathways $(31,32)$. This idea was supported by our finding that $\mathrm{scFv}$ antibodies partially colocalized with ubiquitin and LC3 in Hek293 cells (Figure 3, B and C, and Supplemental 3, C and D).
Moreover, VH1Vk9 and VH7Vk9 scFv antibodies were coimmunoprecipitated with $\mathrm{K} 48$ or K63 polyubiquitinated proteins, which are destined for proteasomal and autophagic degradation, respectively (Supplemental Figure 4A). Using the same immunoprecipitation method, we found that expression of $\mathrm{VH} 7 \mathrm{Vk} 9 \mathrm{scFV}$ stimulated the K63 and K48 polyubiquitination of TDP-43 (Figure 3D). We confirmed the degradative pathways by treating cells with an autophagy inhibitor (bafilomycin) or a proteasome inhibitor (MG-132) (Supplemental Figure 4, B and C). Interestingly, we found that the levels of TDP-43 were increased in the presence of VH1Vk9 and VH7Vk9 scFv antibodies in the cells treated with bafilomycin (Supplemental Figure 4B), and the proteasomemediated degradation of TDP-43 via VH7Vk9 scFv was confirmed by the accumulation of TDP- 43 in the presence of MG-132 (Supplemental Figure 4C). Thus, these results suggest that intracellular $\mathrm{scFv}$ antibodies drive TDP-43 to degradation pathways, leading to a decrease in cytoplasmic TDP-43 levels.

Single-chain antibodies reduced TDP-43 mislocalization and aggregation. To assess the ability of scFv antibodies to reduce TDP43 mislocalization and aggregation, we used a cell model system of TDP-43 proteinopathy that is based on oxidative stress caused by treatment with $50 \mu \mathrm{M}$ ethacrynic acid (EA) in Hek293 cells (33). As shown in Supplemental Figure 5, A-C, treatment of Hek293 cells with EA increased oxidative stress and induced TDP-43 cytoplasmic mislocalization and aggregation. Remarkably, the presence of VH1Vk9 and VH7Vk9 scFv antibodies in transfected cells led to a reduction in cytoplasmic levels of TDP-43 detected by immunofluorescence microscopy (Figure 4A and Supplemental Figure 5D). Moreover, in the insoluble fractions prepared from cells treated with EA, TDP-43 levels were reduced by up to $50 \%$ in cells expressing VH7Vk9 scFv antibody (Figure 4B and Supplemental Figure 5E). 
A
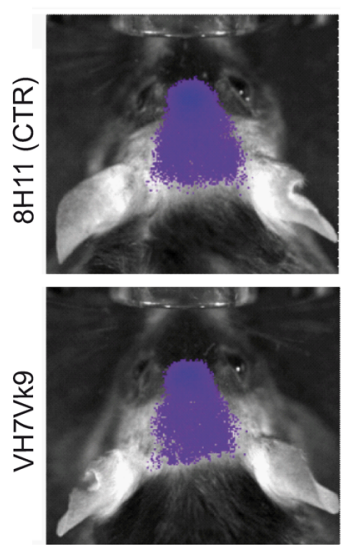

1 day
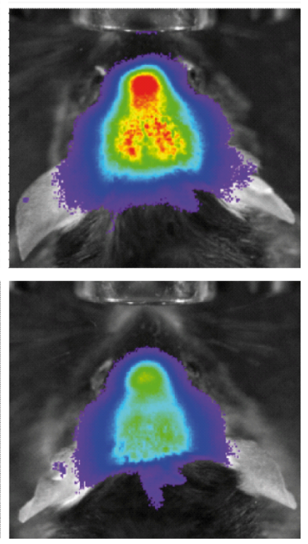

2 days
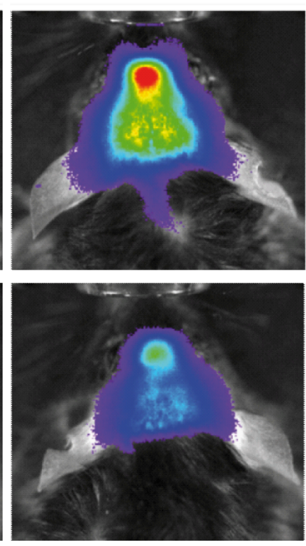

3 days
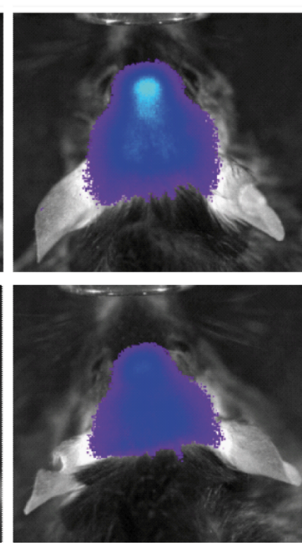

5 days

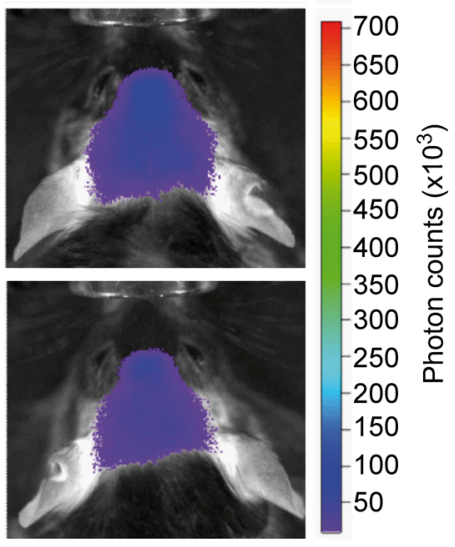

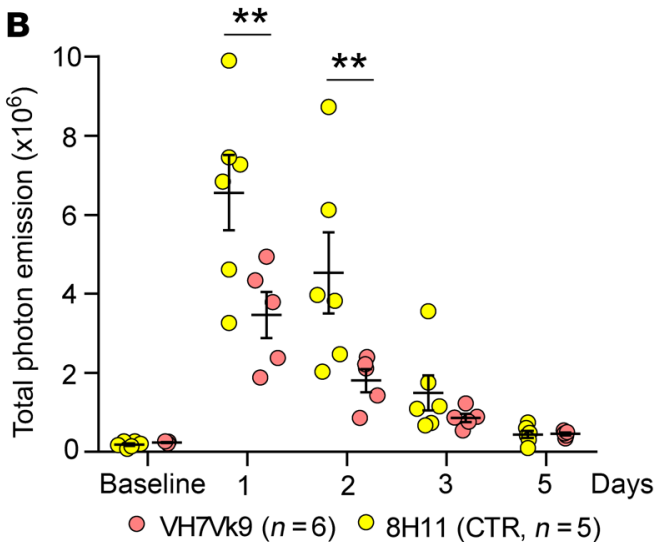
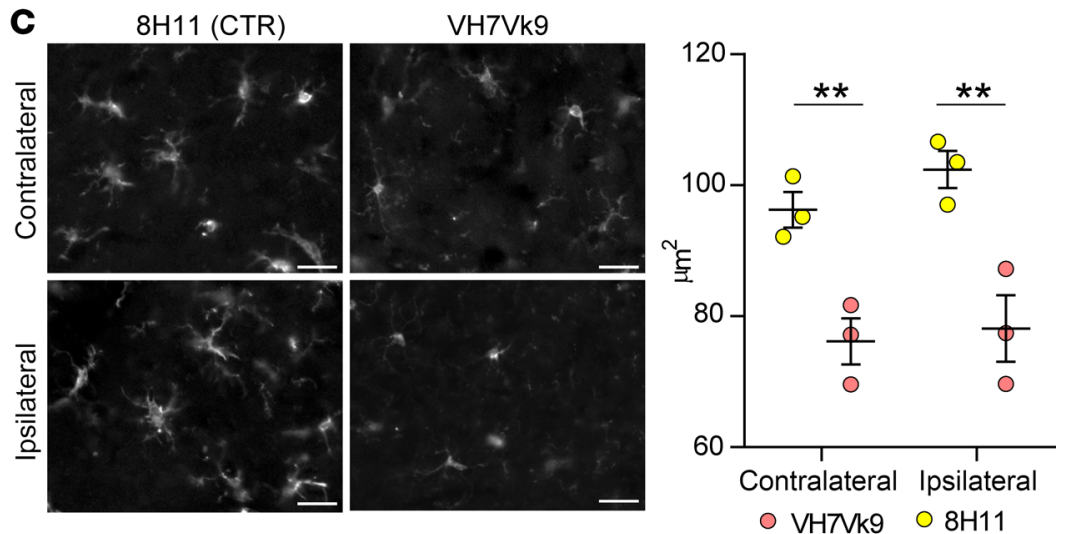

Figure 5. ScFv antibody reduces NF-KB activation in LPS-induced inflammation in vivo. (A) Real-time imaging of TLR2-luciferase response before (baseline) and 1 to 5 days after LPS injection and (B) relative quantification. Images of representative mouse brains previously injected with scAAV2/9 expressing control or VH7Vk9 scFv. Color scale calibration in photon counts (minimum 10,000, maximum $7.08 \times 10^{5}$ ). Graph shows quantification of photon emission. $n=5-6$ individual mice. Two-way, repeated-measures ANOVA $P=0.0004 ;{ }^{*} P<0.01$ versus VH7Vk9, by Sidak's multiple comparisons test. (C) Representative enlarged images of Iba1 staining and quantification of cell body size in contralateral and ipsilateral cortices of mice injected with scAAV2/9 and challenged with LPS. Graph shows quantification of Iba1 ${ }^{+}$cells. $n=3$ individual mice (dots). Two-way ANOVA interaction $P=0.582$, hemisphere $P=0.300$, and scFv $P=0.0003 ;{ }^{* *} P<0.01$, by Bonferroni's multiple comparisons test. Data represent the mean \pm SEM. Scale bars: $25 \mu \mathrm{m}$. CTR, $8 \mathrm{H} 11 \mathrm{scFv}$.

Virus-mediated expression of VH7Vk9 scFv antibody reduced microglial activation and $N F-\kappa B$ activity in an acute model of inflammation. Among the 2 single-chain antibodies, VH7Vk9 was more efficient at reducing $\mathrm{NF}-\kappa \mathrm{B}$ activity and TDP-43 proteinopathy in vitro. We therefore generated a scAAV2/9 viral vector encoding VH7Vk9 scFv (CMV promoter, IgH region and Myc tag included) to further investigate the effect of the antibody in vivo. As a control, we used a scAAV2/9 vector (named $8 \mathrm{H} 11$ ) encoding scFv against GFP.

Virus $\left(1.5 \times 10^{10} \mathrm{vg}\right)$ was injected unilaterally into the cortex of mice ( $n=5$ control mice and $n=6$ VH7Vk9-treated mice; 4 months of age) bearing the TLR2-luciferase transgene, a reporter of microglial activation (34). One month after virus delivery, mice were intraperitoneally injected with LPS, and TLR2-luciferase activity was monitored for 5 days (Supplemental Figure 6A). As shown in Figure 5, A and B, LPS injection induced strong luciferase activity in the brain after 1 and 2 days in control scAAV-treated mice. Remarkably, expression of VH7Vk9 scFv reduced TLR2luciferase activity by almost $50 \%$ compared with activity induced by control $\mathrm{scFv}$ antibody. We repeated the experiment with age- matched nontransgenic mice ( $n=3$ per group) (Supplemental Figure 6B) and analyzed brain samples 24 hours after LPS injection. Quantification of ionized calcium-binding adaptor molecule 1 (Iba1) staining revealed a consistent reduction in microglial body size (Figure 5C) in the brains of mice treated with VH7Vk9 scFv when compared with control $8 \mathrm{H} 11 \mathrm{scFv}$ and a concomitant reduction in total staining (Supplemental Figure 6C). Interestingly, the effect on microglial cells was the same in ipsilateral and contralateral brain hemispheres, suggesting an effect of secreted $s c F v$ antibodies at a long distance from the site of viral injection. We performed a cytokine array on the ipsilateral cerebral cortex of the injected mice. All inflammatory factors significantly different from the control condition were found to be reduced by VH7Vk9 expression (Supplemental Figure 6D).

Virus-mediated expression of VH7Vk9 scFv antibody ameliorated cognitive deficits and TDP-43 pathology in TDP-43 $3^{\mathrm{G} 448 \mathrm{C}}$ mice. We injected scAAV2/9 virus encoding VH7Vk9 or 8 H11 (anti-GFP) $\mathrm{scFv}$ antibodies into transgenic mice expressing genomic fragments encoding human $T D P-43^{\mathrm{G} 348 C}$, a mouse model that exhibits cytoplasmic accumulations of TDP-43 in neurons during aging 
A

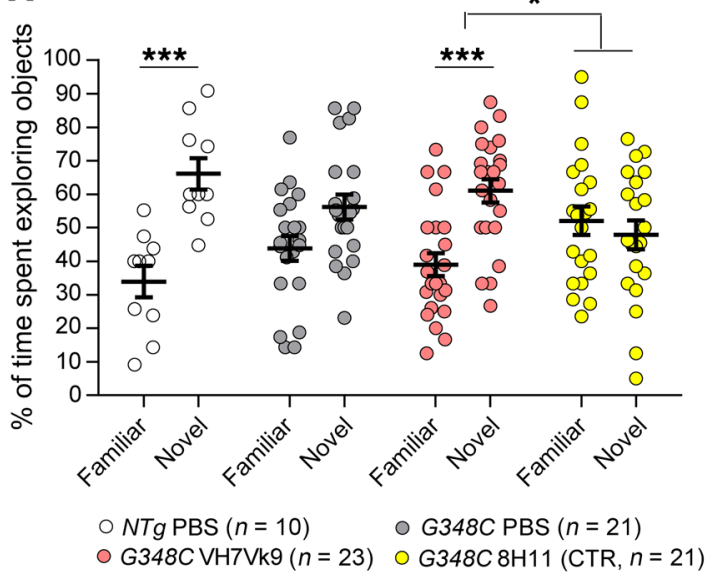

B

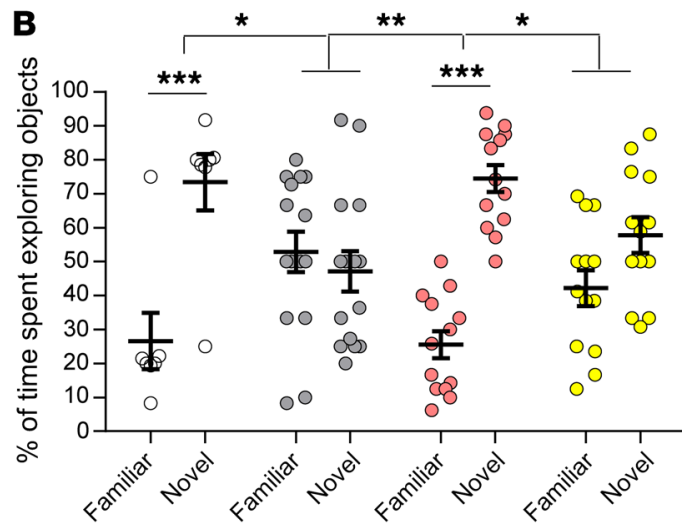

O NTg PBS $(n=7) \quad$ O G348C PBS $(n=15)$

○ G348CVH7Vk9 $(n=13)$ o G348C $8 \mathrm{H} 11(\mathrm{CTR}, n=13)$
C
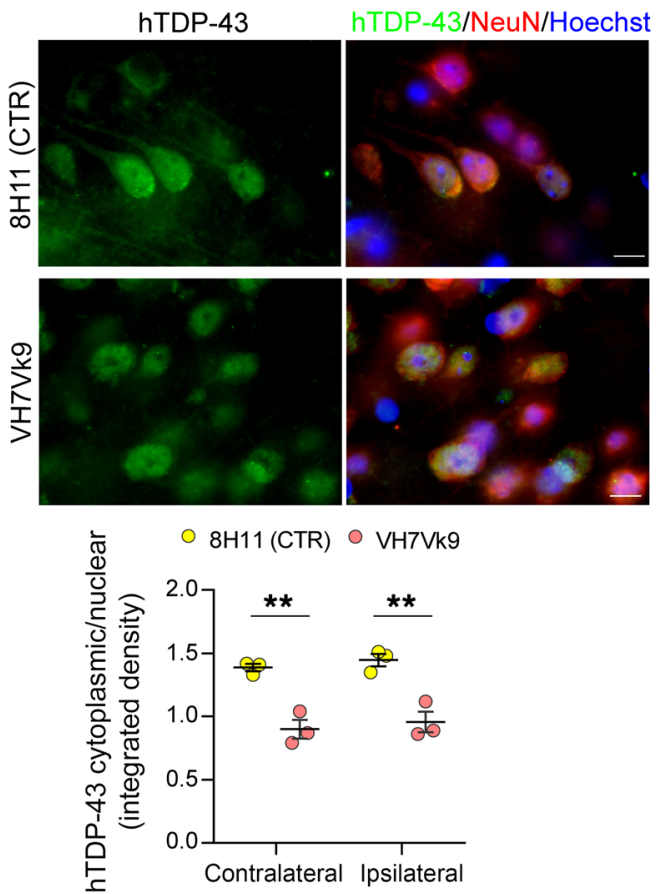

D
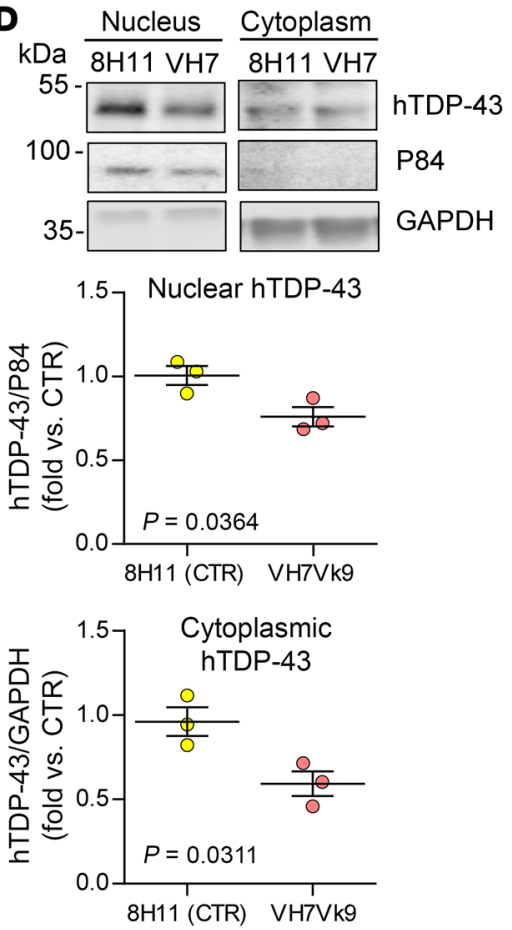

E

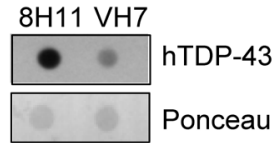

Cytoplasmic insoluble

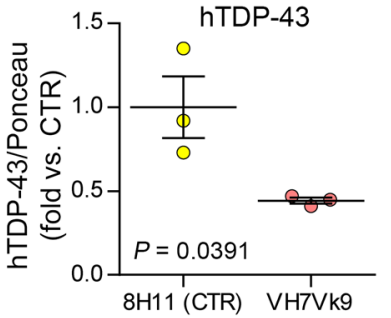

Figure 6. Intracortical injection of scAAV2/9 encoding for scFv antibody improves cognitive deficits and restores TDP-43 mislocalization in TDP-43 $3348 \mathrm{C}$ mice. NOR test 2 months (A) and 4 months (B) after injection. Graphs in $\mathbf{A}$ and $\mathbf{B}$ show the percentage of time spent with objects and the number of individual mice tested (dots). (A) Two-way ANOVA $P=0.0004 ;{ }^{* *} P<0.001$ and ${ }^{*} P<0.05$, by Sidak's multiple comparisons test. (B) Two-way ANOVA $P<0.0001 ;{ }^{* *} P<0.001$ for familiar versus novel object, by Sidak's multiple comparisons test; ${ }^{*} P<0.05$ and ${ }^{* *} P<0.01$ among groups, by uncorrected Fisher's least significant difference (LSD) test. (C) Quantification of hTDP-43 mislocalization in cortical neurons and representative images. Data represent the cytoplasmic to nuclear ratio of TDP-43 integrated density. $n=3$ individual mice (dots). Two-way ANOVA interaction $P=0.979$, hemisphere $P=0.3747$, scFv $P<0.0001 ;{ }^{* *} P<0.01$ versus control scFv, by Sidak's multiple comparisons test. Shown are hTDP-43 (green) and merged images of hTDP-43, NeuN (red), and Hoechst (nuclei, blue). Scale bars: $20 \mu \mathrm{m}$. Representative blots and quantification of nuclear and cytoplasmic (D) and insoluble (E) hTDP-43 in ipsilateral cortices. $n=3$ individual mice (dots). hTDP-43 was normalized to p84 or GAPDH fraction markers (D) and to Ponceau (E). Values are expressed as the fold change versus control scFv. Statistical analysis was done by unpaired $t$ test. Data represent the mean \pm SEM. CTR, $8 \mathrm{H} 11 \mathrm{scFv}$.

(35). Eight-month-old TDP- $43^{\mathrm{G} 348 \mathrm{C}}$ symptomatic mice were injected intracortically and unilaterally with scAAV2/9 viral vectors $(9.2$ $\left.\times 10^{9} \mathrm{vg}\right)$ carrying virus coding for VH7Vk9 $(n=23$ mice: 11 males and 12 females); virus bearing the control $8 \mathrm{H} 11 \mathrm{scFv}$ antibody $(n=$ 23 mice: 11 males and 12 females); or PBS ( $n=24$ mice: 11 males and 13 females). We also injected nontransgenic ( $N T g$ ) mice ( $n=6$ male and 4 females) with PBS as healthy controls for the behavioral tests. The mice were analyzed for cognitive changes at 2 and 4 months after virus injection. Immunohistochemical microscopy with antiMyc antibody confirmed the expression of VH7Vk9 scFv antibody in brain cortex in neurons and microglial cells (data not shown).

Two and four months after injection, the novel object recognition (NOR) test was performed (Figure 6, A and B). The TDP- $43^{\mathrm{G} 348 \mathrm{C}}$ mice (10 or 12 months of age) injected with PBS or control $8 \mathrm{H} 11$ scFv showed memory impairments, as they were unable to discriminate between novel and familiar objects. In contrast, $N T g$ mice and 
A

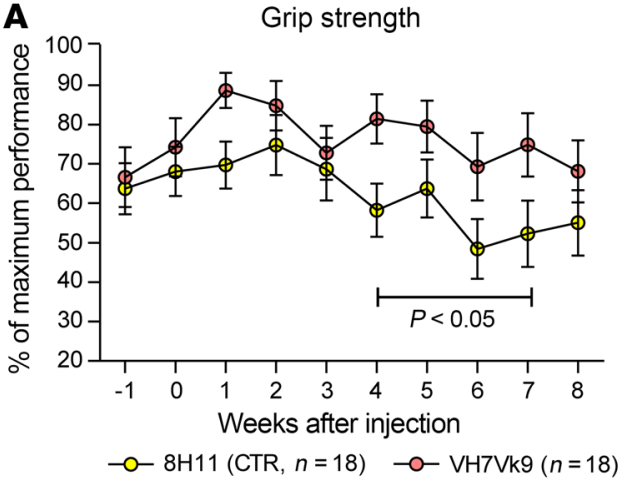

B NMJs in gastrocnemius

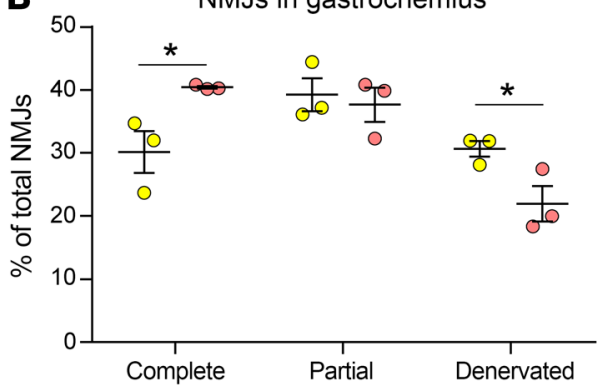

C
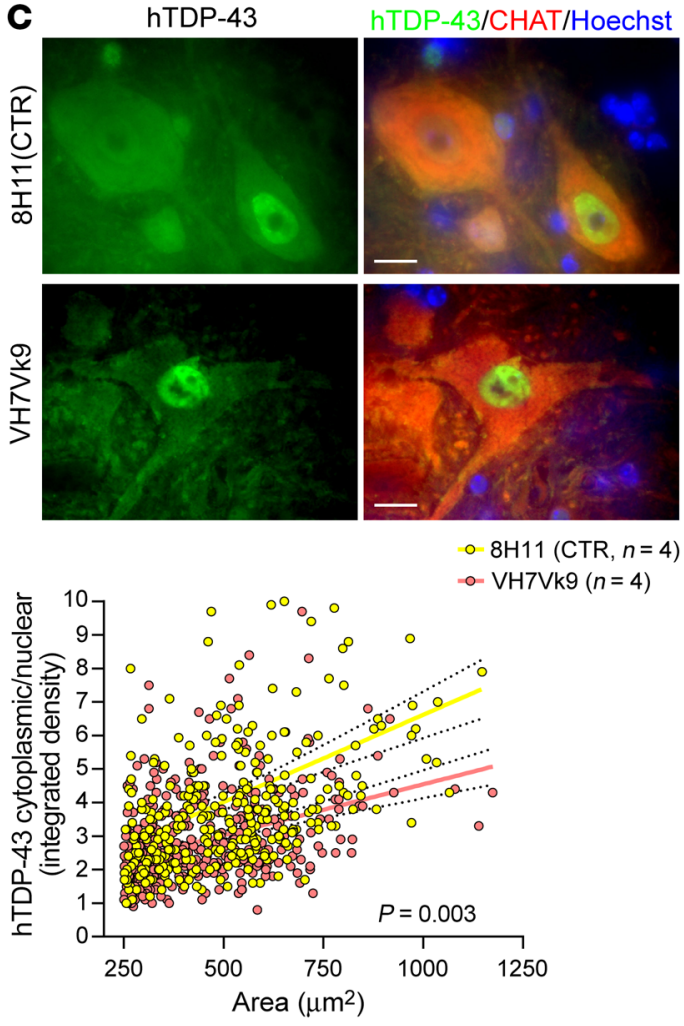

○ $8 \mathrm{H} 11$ (CTR) O VH7Vk9

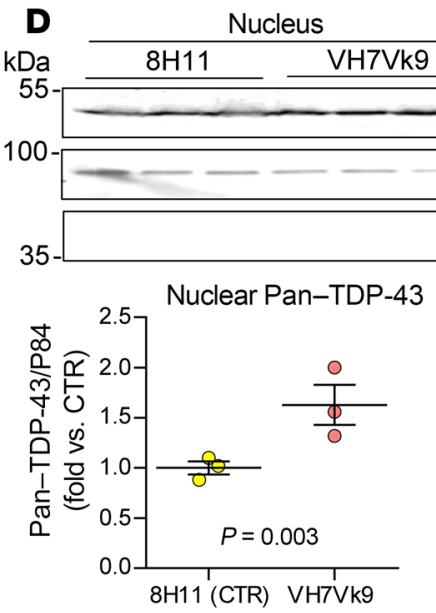

$\mathbf{E}$

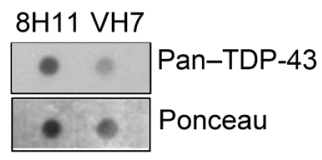

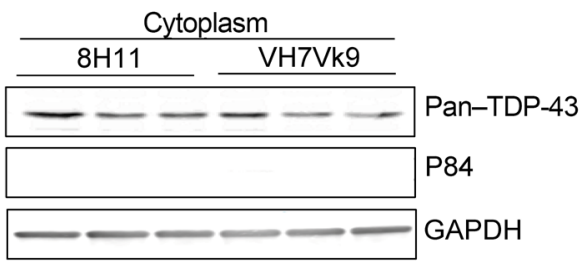
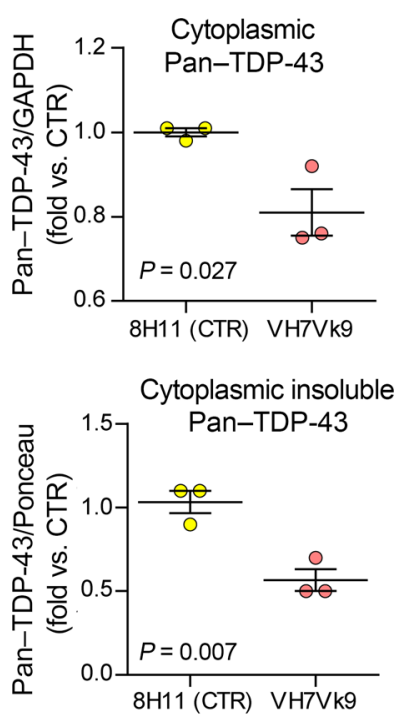

Figure 7. scAAV2/9-mediated delivery of scFv ameliorates motor performance and pathological defects in TDP-43 ${ }^{A 315 T}$ mice. (A) Grip strength analysis showing the percentage of maximum performance of each single mouse and the number of individual mice (dots) analyzed. Two-way ANOVA corrected for repeated-measures interaction $P=0.823$, time $P=0.0512$, scFv $P<0.0001 ;{ }^{*} P<0.05$ versus control scFv, by uncorrected Fisher's LSD. (B) Percentage of total NMJs. $n=3$ individual mice (dots). Two-way ANOVA $P=0.0063$; ${ }^{*} P<0.05$ versus control scFv, by uncorrected Fisher's LSD test. (C) Representative image and quantification of hTDP-43 (green) in motor neurons (CHAT, red) of lumbar spinal cord. The cytoplasmic to nuclear ratio of the TDP-43 integrated density of each cell was plotted according to the area of the cell. Data represent single-cell ratios (dots) analyzed from 4 individual mice. Solid line indicates the linear regression and dotted lines the $95 \% \mathrm{Cls}$. Slope diversity $P=0.0033$. Scale bars: $10 \mu \mathrm{m}$. Representative blots and quantification of nuclear and cytoplasmic (D) and insoluble (E) pan-TDP-43 in lumbar spinal cord from 3 individual mice (dots). TDP-43 was normalized to p84 or GAPDH fraction markers (D) or to Ponceau (E), and values are expressed as the fold change versus control scFv. (B-E) Statistical significance was determined by unpaired $t$ test. Data represent the mean \pm SEM. CTR, $8 \mathrm{H} 11 \mathrm{scFv}$.

TDP-43 ${ }^{\mathrm{G} 348 \mathrm{C}}$ mice expressing VH7Vk9 scFv spent significantly more time with the novel object both 2 months (Figure 6A) and 4 months (Figure 6B) after virus injection, almost matching the results obtained with healthy nontransgenic mice intracortically injected with PBS. We analyzed brain tissue samples ( $n=3$ mice per group) by immunofluorescence microscopy to determine the distribution of human TDP-43 (hTDP-43) in cortical neurons. Neurons were visualized by NeuN staining, and cytoplasmic and nuclear TDP-43 was measured in each cell. The data revealed a reduction in cyto- plasmic staining of hTDP-43 in VH7Vk9-expressing TDP-43 ${ }^{\mathrm{G} 348 \mathrm{C}}$ mice, with an approximately $40 \%$ reduction of the cytoplasmic to nuclear ratio of hTDP-43 in both ipsilateral and contralateral cortical neurons (Figure 6C and Supplemental Figure 7A).

We performed Western blotting on nuclear and cytoplasmic fractions from ipsilateral cortices. As shown in Figure 6D, the analyses confirmed decreased levels of hTDP-43 in the cytoplasmic and nuclear fractions of VH7Vk9-expressing TDP-43 $3^{\mathrm{G} 48 \mathrm{C}}$ mice. We also detected a reduction of insoluble cytoplasmic hTDP- 
A
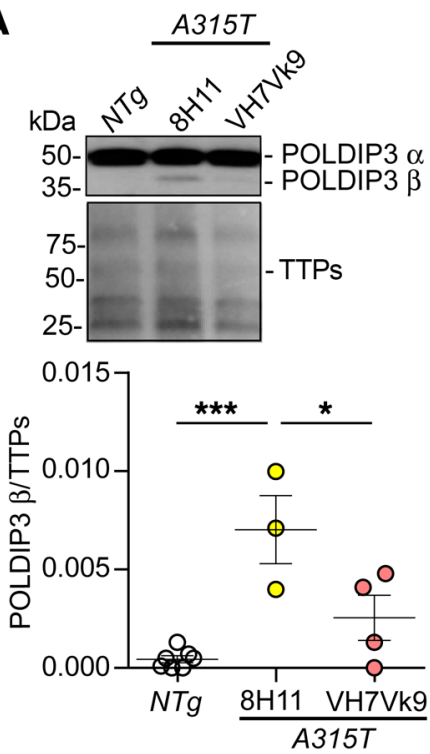

C
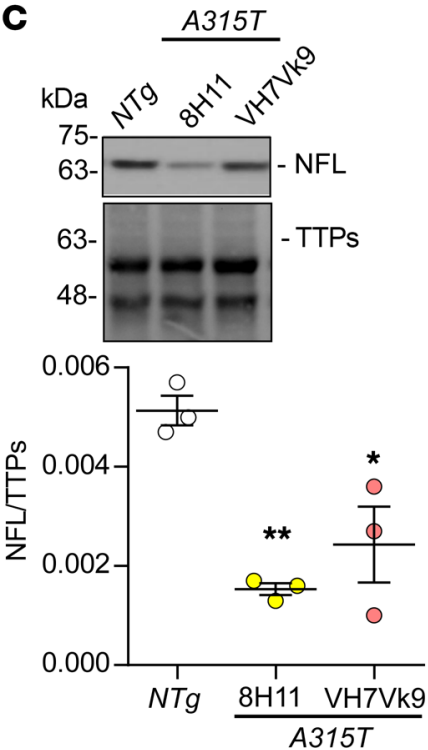

B
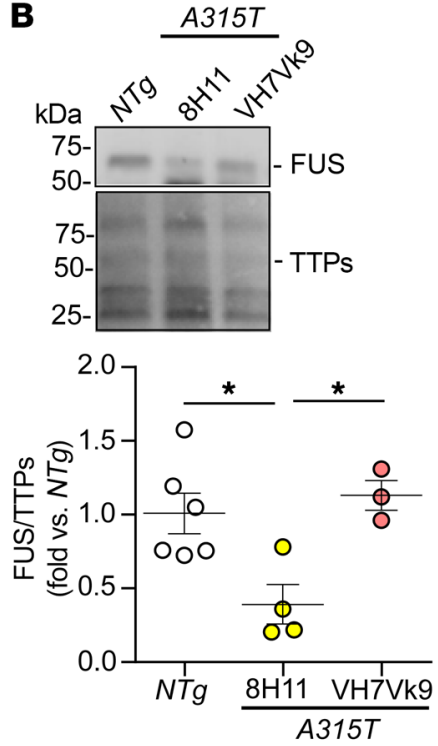

D

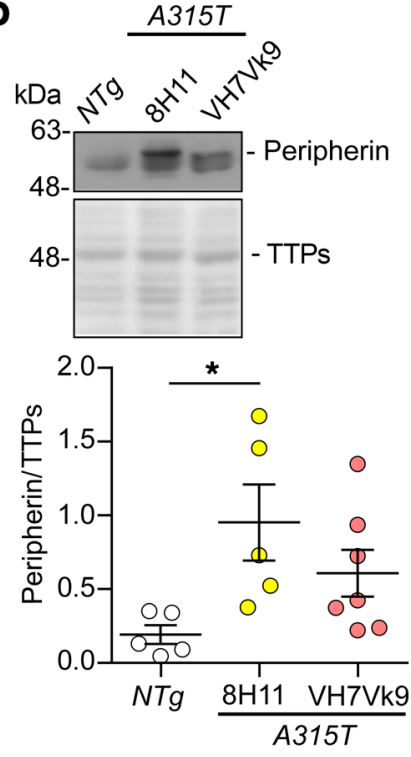

Figure 8. scAAV2/9-mediated delivery of scFv restores TDP-43 functions in the lumbar spinal cord of TDP-43 ${ }^{A 315 T}$ mice. (A) Representative Western blot and quantification of nuclear POLDIP3 splicing in NTg $(n=7)$, A315T scAAV control-treated $(n=4)$, and A315T scAAV-VH7Vk9-treated $(n=4)$ individual mice (dots). One-way ANOVA $P=0.0008$; ${ }^{* *} P<0.001$ and ${ }^{*} P<0.05$, by Tukey's multiple comparisons test. (B) Representative Western blot and quantification of nuclear FUS in NTg $(n=6)$, A315T scAAV control-treated $(n=4)$, and A315T scAAV-VH7Vk9-treated $(n=3)$ individual mice (dots). One-way ANOVA $P=0.0112$; ${ }^{*} P<0.05$, by Tukey's multiple comparisons test. Values are expressed as the fold change versus NTg. (C) Representative Western blot and quantification of cytoplasmic NFL in NTg ( $n=$ 3), A315T scAAV control-treated $(n=3)$, and A315T scAAV-VH7Vk9treated $(n=3)$ individual mice (dots). One-way ANOVA $P=0.0042$; ${ }^{*} P<0.01$ and ${ }^{*} P<0.05$, by Tukey's multiple comparisons test. (D) Representative Western blot and quantification of cytoplasmic peripherin in NTg $(n=5)$, A315T scAAV control-treated $(n=6)$, and A315T scAAV-VH7Vk9-treated $(n=7)$ individual mice (dots). One-way ANOVA $P=0.0392 ;{ }^{*} P<0.05$, by Tukey's multiple comparisons test. Protein levels were normalized to total transferred proteins (TTPs). Data represent the mean \pm SEM. CTR, $8 \mathrm{H} 11 \mathrm{scFv}$.

We injected 8-month-old TDP- $43^{A 315 T}$ mice intrathecally with scAAV2 $/ 9$ virus $\left(5 \times 10^{10} \mathrm{vg}\right)$ expressing VH7Vk9 $(n=18$ mice: 11 females and 7 males) or $8 \mathrm{H} 11$ anti-GFP control scFv $(n=$ 18 mice: 11 females and 7 males). An inverted grid test was carried out to measure muscle strength over a period of 2 months after virus injection and revealed improved performance for VH7Vk9-treated TDP-43 ${ }^{A 315 T}$ mice when compared with scAAV-8H11-injected mice (Figure 7A). No changes were observed in body weight (data not shown). In agreement with the improved motor strength observed, the VH7Vk9-expressing TDP-43 ${ }^{A 315 T}$ mice exhibited better preservation of neuromuscular junctions (NMJs) (Figure 7B), with an increase of completely innervated junctions and a decrease of denervated junctions in the muscles analyzed. We then analyzed the lumbar spinal cords of the injected mice ( $n=4$ mice per group). Our analysis of choline acetyltransferase-positive $\left(\mathrm{CHAT}^{+}\right)$ cells, i.e., motor neurons, revealed no difference in the number of cells (Supplemental Figure 9A), nevertheless, immunofluorescence quantification revealed a significant reduction of 43 (Figure 6E). In addition to neuronal changes in the hTDP-43 pathology, we detected a reduction of microgliosis, observed by Iba1 staining, in both the ipsilateral and contralateral cortices of VH7Vk9 scFv-injected mice (Supplemental Figure 7B).

Finally, 4 months after injection, the open field test (OFT) showed that the VH7Vk9-injected TDP- $43^{\mathrm{G} 34 \mathrm{C}}$ mice had less anxiety (Supplemental Figure 8A). Mice injected with VH7Vk9 were indeed able to remain in the center of the box for a longer period of time than the control scFv TDP- $43^{\mathrm{G} 348 C}$-injected mice and for a duration nearly equivalent to that of nontransgenic mice that were intracortically injected with PBS. The cognitive improvements observed 4 months after injection correlated with reduced levels of insoluble hTDP-43 in the cerebral cortex of VH7Vk9-injected mice (Supplemental Figure 8B).

Virus-mediated expression of VH7Vk9 scFv antibody improved motor performance and reduced TDP-43 pathology in TDP-434315T mice. We also tested the effect of VH7Vk9 $\mathrm{scFv}$ in mice expressing a human genomic transgene encoding the TDP- $43^{A 315 T}$ mutant (35). cytoplasmic hTDP-43 (Figure 7C and Supplemental Figure 9, B and C). A correlation between TDP- 43 mislocalization and cell body size revealed that larger cells were more sensitive to TDP-43 proteinopathy (Figure 7C), however, the slope of the linear regression of TDP43 mislocalization in motor neurons of scAAV-VH7Vk9-injected mice was significantly reduced compared with that of scAAV-8H11injected mice $(Y=0.0052 \times X+1.409$ for control motor neurons and $Y=0.0030 \times X+1.505$ for VH7Vk9 motor neurons), confirming a reduction of TDP-43 mislocalization mediated by VH7Vk9.

Western blots of cytoplasmic and nuclear TDP- 43 fractions showed a significant decrease of cytoplasmic TDP-43 in lumbar spinal cords of mice injected intrathecally with scAAV-VH7Vk9 (Figure 7D). Moreover, dot blot analyses of cytoplasmic insoluble TDP-43 revealed a significant decrease of aggregated/insoluble pan-TDP-43 in VH7Vk9-treated mice (Figure 7E).

We then analyzed the activation of NF- $\mathrm{\kappa B}$ and inflammation in the lumbar spinal cords. Western blot analysis of cytoplasmic total p65 showed no differences, whereas a significant decrease of 
the activated and phosphorylated subunit of NF- $\mathrm{B}$ was observed in VH7Vk9-treated mice (Supplemental Figure 10A). We observed a concomitant reduction of the glial fibrillary acidic protein (GFAP) and Iba1 markers, suggesting decreased neuroinflammation (Supplemental Figure 10A), which was further confirmed by immunofluorescence analyses. Iba1 and GFAP staining was indeed reduced in VH7Vk9-treated mice, in which both microglia and astrocytes had smaller body sizes (Supplemental Figure 10, B and C). Next, we performed a cytokine array on spinal cord and detected a general downregulation of inflammatory cytokines in the scAAV-VH7Vk9-injected mice (Supplemental Figure 10D).

Finally, we examined whether treatment with scFv VH7Vk9 antibody in the spinal cord of TDP- $43^{A 315 T}$ mice was able to restore proper functionality of the protein (Figure 8). We performed Western blotting to analyze the splicing of DNA polymerase delta-interacting protein 3 (POLDIP3) (36) as well as the regulation of RNA-binding protein FUS (37) and light-chain neurofilament protein (NFL) (38), three proteins whose RNAs are regulated by TDP-43 in the nucleus or cytoplasm (Figure $\left.8, \mathrm{~A}^{-} \mathrm{C}\right)$. We found that, in TDP- $43^{A 315 T}$ mice, POLDIP3 was aberrantly spliced, whereas FUS and NFL levels were downregulated compared with levels in NTg mice, confirming the alteration of TDP43 functions. In spinal cord samples from $T D P-43^{A 315 T}$ mice injected with VH7Vk9, we observed a partial restoration of normal levels of these 3 different proteins, close to the levels detected in the nontransgenic mice. We also examined the levels of peripherin, another pathological marker of ALS, which is abnormally upregulated in $T D P-43^{A 315 T}$ mice (35). Expression of VH7Vk9 antibodies produced a downregulation of peripherin levels in the spinal cords of TDP-43 $3^{A 315 T}$ mice (Figure 8D). Thus, the combined results show that expression of VH7Vk9 antibodies reestablished the normal functions of TDP-43 in the TDP- $43^{A 315 T}$-transgenic mouse model.

\section{Discussion}

Single-chain antibodies, a new tool in nanomedicine, have been recently introduced to the field of neurodegenerative diseases through the generation of a variety of recombinant antibodies that bind to various epitopes of pathological proteins implicated in $\mathrm{AD}, \mathrm{PD}, \mathrm{HD}$, and prion disease (39) and through the preclinical demonstration of their efficacy in ameliorating pathological symptoms. Some single-chain antibodies are now available for ALS (25-27), but only the scFv targeting misfolded superoxide dismutase 1 (SOD1) proved to be effective in vivo in ameliorating pathological changes and slowing disease progression in a mouse model with an ALS-linked SOD1 mutation (26, 40, 41).

Here, we report for the first time to our knowledge the generation of a novel scFv antibody against TDP-43, named VH7Vk9, and its therapeutic effect when delivered in mouse models of ALS/FTD with TDP-43 pathology. The VH7Vk9 scFv antibody specifically targeted the RRM1 domain of TDP-43, a region implicated in the cytosolic mislocalization and aggregation of the protein $(11,12)$ and in the interaction with the p65 NF-кB subunit (13). The VH7Vk9 antibody was designed with a secretion signal peptide to allow the antibody to also be effective on distant cells. We demonstrated that virus-mediated delivery of VH7Vk9 in the CNS of transgenic mice expressing mutant hDP-43 succeeded in ameliorating cognitive and motor deficits as well as in reducing TDP43 proteinopathy and neuroinflammatory changes.
It is now well established that the cytoplasmic accumulation of TDP-43 can be cytotoxic $(8,42)$. Both mRNA and protein levels of TDP-43 were found to be increased in CNS tissues (13) and peripheral cells (14) of patients with sALS. The factors triggering the initial mislocalization of TDP-43 in pathologies are largely unknown. Yet a reduction of cytoplasmic TDP-43 levels can rescue neurons from death (42-45). TDP-43 can be degraded through proteasomal and autophagic pathways (32). There is evidence that both of these pathways can act on the soluble TDP-43 present in the cytoplasm, with the proteasome contributing to the degradation of monomers and autophagy promoting the degradation of early oligomers, microaggregates, or late macroaggregates (32). Previous studies have established the therapeutic potential of autophagy enhancers in clearing TDP-43 from the cytoplasm $(46,47)$. Here, we showed that the scFv VH7Vk9 antibody against the RRM1 domain of TDP43 can enhance the Lys- 48 and Lys- 63 polyubiquitin chains bound to TDP-43, thereby enhancing protein degradation toward the proteasomal and autophagic pathways (Figure3).

There are previous reports of $\mathrm{scFv}$ antibodies able to decrease the levels of target proteins in other neurodegenerative disorders. This can be achieved by inserting a specific signal peptide for degradation in the $\mathrm{scFv}$ constructs $(21,24,27)$. In contrast, we show here that a $\mathrm{scFv}$ without signal peptide for degradation can help to drive target proteins to degradation pathways by enhancing their polyubiquitination. We can speculate that the $\mathrm{scFv}$ antibody against the RRM1 domain may interfere with TDP-43 aggregation and increase the soluble TDP- 43 forms that might be more suitable for polyubiquitination. Alternatively, the $\mathrm{scFv}$ antibody may form complexes with TDP-43, which in turn may become more prone to polyubiquitination. In any case, the observed reduction of TDP-43 levels mediated by the VH7Vk9 scFv antibody appeared to occur at the protein degradation level. As shown in Supplemental Figure 1E, the VH7Vk9 scFv antibody did not interfere with the binding of TDP-43 to its own RNA in Hek293 cells, and therefore it does not affect TDP-43 autoregulation via mRNA binding (28). Our cell culture studies also revealed that the $\mathrm{scFv}$ antibodies can alleviate mislocalization and aggregation of TDP-43 under conditions of oxidative stress (Figure 4). Therefore, it is possible that the scFv antibody also protects the TDP-43 RRM1 domain from oxidative damage and misfolding, which can trigger protein aggregation $(11,12)$. Interestingly, the reduction of TDP-43 levels mediated by VH7Vk9 was found to be safe for cultured cells in vitro (Supplemental Figure 3A) as well as for motor neurons in TDP-43 ${ }^{A 315 T}$ mice (Supplemental Figure 9A). Our studies with TDP-43-transgenic mouse models revealed an antibody-mediated reduction of cytoplasmic and aggregated TDP-43 levels in neurons (Figure 6, C-E, and Figure 7, C-E) that was associated with a concomitant restoration of cognitive (Figure 6, A and B, and Supplemental Figure $8 \mathrm{~A}$ ) and motor functions (Figure 7A), together with an alleviation of NMJ loss (Figure 7B). We also observed that the presence of VH7Vk9 restored normal levels of proteins such as POLDIP3, FUS, NFL, and peripherin that are aberrantly deregulated in the context of cytoplasmic TDP-43 pathology (Figure 8).

Another therapeutic effect observed with the anti-RRM1 scFv antibody was the reduction of NF- $\kappa \mathrm{B}-$ mediated inflammation. By blocking the interaction of TDP-43 with p65 (Figure 2A), the $\mathrm{scFv}$ antibody was able to reduce $\mathrm{NF}-\mathrm{\kappa B}$ activation in microglial 
cells (Figure 2B). In mouse models with LPS-induced inflammation (Figure 5) or TDP-43 mutations (Supplemental Figure 7B and Supplemental Figure 10B), viral delivery of VH7Vk9 resulted in reduced microgliosis and levels of phosphorylated p65. As a further confirmation of VH7Vk9-mediated NF- $\mathrm{B}$ inhibition, we observed a downregulation of different inflammatory factors in tissues treated with the virus encoding for the scFv antibody (Supplemental Figure 6D and Supplemental Figure 10D). Most of these factors are relevant to ALS. Indeed, studies on cerebrospinal fluid (CSF), plasma, or serum from patients with ALS revealed an upregulation of granulocyte CSF (GCSF) and granulocyte-macrophage CSF (GM-CSF) (48), IFN- $\gamma$ (48), IL-12 (49), IL-17 (48, 50), TNF- $\alpha$ and receptors (51), Rantes (52), monocyte chemoattractant protein 1 (MCP1) (48), and macrophage inflammatory protein 1 (MIP1) $(48,53)$.

It should be noted that the inhibition of NF- $\mathrm{B}$ signaling by VH7Vk9 antibodies is partial and occurs in the context of TDP-43 pathology in neurons or in the context of innate immune activation (such as with LPS) in microglial cells. A previous study (13) showed that TDP-43 can act as a coactivator of $\mathrm{p} 65$ and that overexpression of TDP-43 can enhance NF- $\kappa$ B activation. The blocking of TDP-43 interaction with p65 NF- $\kappa \mathrm{B}$ is beneficial rather than toxic. First, the results presented in Supplemental Figure 2B revealed that attenuation of NF- $\kappa \mathrm{B}$ activity by $\mathrm{scFv}$ antibodies in microglial cells (BV2 cells) challenged by LPS confers protection to N2A neuronal cells exposed to media from LPS-treated BV2 cells. Second, TLR2luciferase mice injected in the brain with an adeno-associated virus (AAV) vector encoding VH7Vk9 scFv antibody remained healthy, and the presence of VH7Vk9 scFv antibodies attenuated microglial activation induced by LPS treatment (Figure 5). Third, expression of scFv antibodies in transfected Hek293 cells did not affect cell survival (Supplemental Figure 3A), nor did it reduce the number of motor neurons in TDP- $43^{A 315 T}$ mice (Supplemental Figure 9A). So far, we have obtained no evidence that VH7Vk9 scFv antibodies cause cellular toxicity. On the contrary, the VH7Vk9 antibody can alleviate mislocalization and aggregation of TDP-43 (Figure 6 and Figure 7), and it can restore normal levels of proteins that are aberrantly regulated in TDP-43 pathology (Figure 8).

It is worth highlighting the fact that our $\mathrm{scFv}$ was designed with a secretion signal, which allowed the antibody to act not only on virus-infected cells but also on distal cells from the injection site. It is indeed remarkable that a reduction of microgliosis in TDP$43^{\mathrm{G} 348 \mathrm{C}}$ and LPS-challenged mice was observed not only in the injected ipsilateral hemisphere but also in the contralateral one. Similarly, the reduction in TDP-43 mislocalization was detected in the ipsilateral and contralateral cortical neurons of mice injected with $T D P-43^{G 348 C}$. The diffusion of $s c F v$ antibodies in the CNS may explain the improved cognitive performance in VH7Vk9-injected mice after a focal intracortical injection of viral vectors.

In conclusion, we demonstrate for the first time to our knowledge that a single-chain antibody against the RRM1 domain of TDP-43 is an efficient therapeutic approach to target TDP-43 proteinopathy and ameliorate cognitive and motor deficits in transgenic mouse models. The target specificity and therapeutic effects of the scFv antibody support the feasibility of developing immunotherapeutic approaches to target intracellular TDP-43 aggregates and neuroinflammation, two hallmarks of ALS and FTD.

\section{Methods}

Anti-TDP-43 antibody, scFv plasmid, and virus production. The BamH1Not1 411-bp fragment was PCR amplified from a previously produced vector containing the full-length hTDP-43 cDNA (13) using the primers TDP-43 BamH1 (5'-GGGGGATCC ACCATGCGCTACAGGAATCCAGT-3') and TDP-43 Not1 (5'-GGGGCGGCCGCTCATTTGCAGTCACACCAT C-3') and subcloned into the pGEX-6P-1 vector (GE Healthcare). This recombinant plasmid was used to transform BL21-competent (DE3-competent) E. coli cells for expression of the 136-aa protein encoding a portion of the N-Terminal region, the nuclear localization signal (NLS), and the entire RRM1 domain of hTDP-43. The 15.68-kDa recombinant protein was subsequently purified using the glutathione S-transferase (GST) gene fusion system (GE Healthcare). From the recombinant protein, MediMabs produced different hybridoma cell lines.

Single-chain antibodies were produced as previously described (26). The pscFv9 plasmid generated contained a CMV promoter, a murine Ig H-secretory signal for efficient folding and secretion, and a human c-Myc epitope to facilitate detection. The control single-chain antibodies D1.3 anti-chicken lysozyme (26) or 8H11 antiGFP (produced in-house) were produced in the same way. To produce scAAV2/9 (AAV2 terminal repeats and AAV9 capsid) encoding scFv, we followed a previously described protocol (26). Briefly, the fragment encoding for the secreted scFv was first HindIII and EcoRV excised, subcloned into the plasmid BluescriptII KS(+) (Stratagene) and then the excised SalI/NotI, and subcloned into the XhoI/NotI-digested plasmid AAV-CMV-GFP (54) replacing the EGFP-encoding sequence. scAAV recombinant viruses were produced in the Hek293T cells cultured in DMEM (Gibco, Thermo Fisher Scientific) supplemented with $10 \%$ normal bovine serum, $100 \mathrm{U} / \mathrm{ml}$ penicillin $\mathrm{G}$, and $100 \mu \mathrm{g} / \mathrm{ml}$ streptomycin. Cotransfection was done with scAAV-scFv, pXR1, and pxx-6 as packaging and helper plasmids, respectively $(54,55)$.

Animal injections, imaging, and tissues collection. For intracortical injections of scAAV2/9, the mice were anesthetized with $2 \%$ isoflurane during the entire procedure and received a unilateral intracortical injection of scAAV2/9 $\left(9.2 \times 10^{9} \mathrm{vg}\right.$ for TDP- $43^{\mathrm{G} 348 \mathrm{C}}$ and $1.5 \times 10^{10}$ vg for TLR2-luciferase) or PBS solution. Mice were placed in a stereotaxic apparatus (David Kopf Instruments), and the primary frontal cortex was then reached $(-1.75 \mathrm{~mm}$ lateral, $+1.0 \mathrm{~mm}$ anteroposterior and $-1.25 \mathrm{~mm}$ dorsoventral to the bregma) with a 33-gauge stainless steel cannula (Plastics One) that was connected to a $25-\mu$ l Hamilton syringe with intramedic polyethylene tubing (PE-50, Clay Adams). A maximum volume of $2 \mu \mathrm{l}$ was infused over a 2 -minute period by means of a microinjection pump (Razel Scientific Instruments, model A-99). Intrathecal injection of scAAV2/9 into TDP-43 $3^{A 315 T}$ mice was performed as previously described (26). A maximum volume of $10 \mu \mathrm{l}$ $\left(5 \times 10^{10} \mathrm{vg}\right)$ was injected with a Hamilton syringe.

Bioluminescence imaging was performed in TLR2-luciferase mice over a period of 5 days after intraperitoneal injection of $5 \mathrm{mg} / \mathrm{kg}$ LPS as previously described (34).

Tissue collection was done after deep anesthesia of the mice with $10 \mu \mathrm{l} / \mathrm{g}$ pentobarbital $12 \mathrm{mg} / \mathrm{ml}$. Mice were perfused transcardially with PBS followed by $4 \%$ paraformaldehyde (PFA) solution in PBS. Brain, spinal cord, and gastrocnemius muscle were rapidly removed, postfixed overnight in 4\% PFA, and transferred into $30 \%$ sucrose in PBS until they sank. Tissues for protein analyses were rapidly removed from the anesthetized mice and frozen on dry ice before storage at $-80^{\circ} \mathrm{C}$. 
Behavioral analyses. Starting 1 week before the virus injection and throughout the treatment period, mutant $T D P-43^{A 315 T}$ mice were subjected once a week to an inverted grid test to monitor their grip strength (56). Briefly, during the test, the mice were allowed to hang on an upside-down grid for a maximum of 90 seconds, and the latency to fall from the grid was considered for analyses. The body weight of the mice was also measured in each session. TDP- $43^{\mathrm{G} 348 \mathrm{C}}$ mice were subjected to novel object recognition (NOR) to test their memory and to an OFT for anxiety at established time points. NOR was done as a 3 -day test, as previously described (57), in a $20 \times 50 \times 30 \mathrm{~cm}$ Plexiglas box for 5 minutes per session. The OFT was performed in a single session. Mice were allowed to discover and move freely in a computermonitored box (58), and the time spent in the center of the box over a total period of 30 minutes was considered for analyses.

Cell transfection and treatments. Mouse microglia (BV2), Hek293, Hek293T, and mouse brain neuroblastoma Neuro-2a (N2A) cell lines were purchased from the American Type Culture Collection (ATCC). All cell lines were maintained in DMEM supplemented with $1 \%$ sodium pyruvate (Gibco, Thermo Fisher Scientific) and $1 \%$ penicillin $G$ and streptomycin (Gibco, Thermo Fisher Scientific) with 10\% FBS (Gibco, Thermo Fisher Scientific). The murine microglial cell line BV2 p65luc (29) was continuously selected and maintained with $100 \mu \mathrm{g} / \mathrm{ml}$ hygromycin (MilliporeSigma).

When $60 \%$ to $70 \%$ confluence was attained, the cells were transfected for 48 hours with Lipofectamine (Invitrogen, Thermo Fisher Scientific) or Jet Prime (Polyplus) transfection reagents according to the manufacturer's instructions.

After 32 hours of transfection, Hek293 cells were treated overnight with $50 \mu \mathrm{M}$ ethacrynic acid (MilliporeSigma) or the same volume of DMSO (MilliporeSigma) . Forty-four hours after transfection, BV2 cells were treated for 4 hours with $500 \mathrm{ng} / \mathrm{ml}$ LPS (MilliporeSigma) or PBS (Gibco, Thermo Fisher Scientific).

Media of transfected BV2 cells were collected after 4 hours of LPS/PBS treatment, centrifuged at $400 \mathrm{~g}$ for 5 minutes to eliminate dead cells and debris, and added to N2A cells seeded the day before.

Untransfected BV2 cells were treated with $5 \mu \mathrm{g} / \mathrm{ml}$ purified scFv for 2 or 6 hours and then treated for 4 hours with $500 \mathrm{ng} / \mathrm{ml} \mathrm{LPS} \mathrm{for} \mathrm{a}$ total of 6 or 10 hours of treatment with scFv. Before the experiments, cells were washed 3 times with PBS to avoid detection of scFv bound to the cell membrane.

For pulse-chase experiments, after 48 hours of transfection with scFv antibodies, Hek293 cell medium was replaced with OptiMEM (Gibco, Thermo Fisher Scientific) containing $10 \mu \mathrm{g} / \mathrm{ml}$ cycloheximide (MilliporeSigma) and $300 \mathrm{nM}$ bafilomycin (MilliporeSigma) or $0.5 \mu \mathrm{M}$ MG-132 (MilliporeSigma) and treated for 3 or 6 hours.

For immunofluorescence, cells were seeded on coverslips. After transfection or treatment, cells were washed in PBS and fixed for 30 minutes with $4 \%$ PFA.

The UV-CLIP procedure, as well as cell survival, luciferase, and DCHFDA assays are described in the Supplemental Methods.

$s c F v$ purification. $s c F v$ antibodies were purified from the medium of Hek293 cells transfected for 48 hours in Opti-MEM (Gibco, Thermo Fisher Scientific). The medium was collected and centrifuged at 400 $g$ for 5 minutes to eliminate dead cells and debris and then stored at $-80^{\circ} \mathrm{C}$. scFv was immunoprecipitated from $10 \mathrm{ml}$ medium overnight with $400 \mu$ l Myc-tagged agarose beads (25\% slurry, Thermo Fisher Scientific). The next day, the beads were spun down by centrifugation at $2500 \mathrm{~g}$ for 5 minutes, moved to columns provided with the kit, and washed twice with TBS and 10-second spins. A double elution was performed by competition with $500 \mu \mathrm{l}$ c-Myc peptide (Gene Script) at $0.5 \mathrm{mg} / \mathrm{ml}$ concentration for 2 hours of rotation at room temperature. Unbound c-Myc peptide was removed by a $5-\mathrm{kDa}$ cutoff spin column (Bio-Rad) and centrifugation (5 min at 15,000 g). Purified scFv antibodies were quantified using an Eon Plate Reader (BioTek).

Human recombinant TDP-43 protein production. The recombinant plasmid expressing the hTDP-43 cDNA (13) was used to transform competent cells of the E. coli host BL21 (DE3) for the expression of total hTDP-43. After cell lysis, the $43-\mathrm{kDa}$ recombinant protein was purified using the GST gene fusion system (GE Healthcare).

ELISA. ELISA assays were performed with an ELISA kit (Peprotech). Different concentrations of TDP-43-GST-tag (made in-house) or BSA (BioBasic) as a control were diluted in PBS, and $100 \mu \mathrm{l}$ was loaded onto an ELISA plate and incubated overnight at room temperature. The following day, the wells were washed 4 times with washing buffer and incubated for 2 hours with $0.2 \mu \mathrm{g} / \mathrm{ml}$ p65-His-tag (Enzo Life Sciences, ALX-201-284) in PBS, $0.4 \mu \mathrm{g} / \mathrm{ml}$ monoclonal antibodies (MédiMabs), $0.4 \mu \mathrm{g} / \mathrm{ml}$ polyclonal anti-TDP-43 (Proteintech), and BSA - all diluted in PBS or directly diluted in medium of transfected cells. The wells were then incubated for 2 hours with rabbit polyclonal anti-His-tag 6X-HRP-conjugated antibody (rabbit polyclonal, Abcam ab1187; 1:10,000). Chemiluminescence was read at $450 \mathrm{~nm}$ using an EnSpire 2300 Multilabel Reader (PerkinElmer).

Cell protein extraction methods. To obtain nuclear and cytoplasmic fractions, cells were collected in cold PBS and centrifuged at $400 \mathrm{~g}$ for 5 minutes. Pellets were lysated in hypotonic buffer $(10 \mathrm{mM}$ HEPES, pH 8, $10 \mathrm{mM} \mathrm{KCl,} 1.5 \mathrm{mM} \mathrm{MgCl}, 1$ mM NaF, $0.5 \mathrm{mM}$ PMSF supplemented with protease and phosphatase inhibitors) and incubated on ice for 15 minutes. NP-40 (0.5\%) was added, and lysates were vortexed and centrifuged for 10 minutes at $900 \mathrm{~g}$. Supernatant was collected as the cytoplasmic fraction. Pellets were then solubilized in extraction buffer (20 mM HEPES, pH 8, $420 \mathrm{mM} \mathrm{NaCl}, 1.5$ $\mathrm{mM} \mathrm{MgCl}$, $1 \mathrm{mM} \mathrm{NaF}, 0.2 \mathrm{mM}$ EDTA, 25\% glycerol, $0.5 \mathrm{mM}$ PMSF supplemented with protease and phosphatase inhibitors), incubated on ice for 15 minutes, sonicated, and centrifuged at 15,000 $g$ for 10 minutes. Supernatant was collected as the nuclear fraction. Total proteins from cells were obtained by lysis in extraction buffer supplemented with $0.5 \% \mathrm{NP}-40$ if immunoprecipitation experiments were planned, or in boiled 1\% SDS followed by centrifugation at $15,000 \mathrm{~g}$ for 10 minutes. RIPA soluble and insoluble proteins were obtained as previously described (57).

For Western blot analysis of cellular media, proteins were precipitated with 4 volumes of cold acetone at $-80^{\circ} \mathrm{C}$ overnight and pelleted by centrifugation at $15,000 \mathrm{~g}$ for 20 minutes. Pellets were resuspended directly in loading buffer.

Proteins were extracted from animal tissues. To obtain the nuclear and cytoplasmic fractions, tissues were lysated in hypotonic buffer (10 mM HEPES-KOH, pH 7.6, $10 \mathrm{mM} \mathrm{NaCl}, 1$ mM KH $\mathrm{PO}_{4}, 5$ $\mathrm{mM} \mathrm{NaHCO}, 5 \mathrm{mM}$ EDTA, pH 8, $1 \mathrm{mM} \mathrm{CaCl}, 0.5 \mathrm{mM} \mathrm{MgCl}_{2}$ supplemented by protease and phosphatase inhibitors), homogenized, and solubilized by rotation at $4^{\circ} \mathrm{C}$ for 10 minutes, followed by addition of $2.5 \mathrm{M} 10 \%$ sucrose and centrifugation for 10 minutes at 6300 g. Supernatant was collected as the cytoplasmic fraction. Pellets were resuspended in extraction buffer (10 mM Tris, pH 7.4, $300 \mathrm{mM}$ sucrose, $1 \mathrm{mM}$ EDTA, $0.1 \% \mathrm{NP}-40$ supplemented by protease and 
phosphatase inhibitors), homogenized, and centrifuged for 5 minutes at $4000 \mathrm{~g}$. The pellets were washed 3 times with extraction buffer followed by centrifugations. After the last centrifugation, the pellets were resuspended in RIPA buffer (50 mM Tris, pH 7.4, 1 mM EDTA, $150 \mathrm{mM} \mathrm{NaCl}, 1 \% \mathrm{NP}-40$ supplemented with $2 \%$ SDS and protease and phosphatase inhibitors) and sonicated. To obtain insoluble proteins from the cytoplasm, $1 \%$ Triton X-100 was added to the cytoplasmic fraction, sonicated, and ultracentrifuged at 107,000 $\mathrm{g}$ for $30 \mathrm{~min}$ utes. Supernatant was collected as the cytoplasmic soluble portion, whereas pellets were resuspended in RIPA buffer and sonicated, as with nuclear pellets.

Proteins were quantified using the Lowry method (Bio-Rad) or the bicinchoninic acid (BCA) method (Bio-Rad) when SDS was present in the lysate. Western blotting and dot blot methods and cytokine array procedures are described in the Supplemental Methods.

Immunoprecipitation. Immunoprecipitation of TDP-43 was done by coating $40 \mu$ l Dynabeads (Invitrogen, Thermo Fisher Scientific) with $2 \mu \mathrm{g}$ rabbit polyclonal anti-TDP-43 antibody (Proteintech) for 2 hours at room temperature on rotation. The antibody was cross-linked to the beads by a 30-minute incubation, rotating with $20 \mathrm{mM}$ dimethyl pimelimiolate dihydroxichloride (MilliporeSigma) in $0.2 \mathrm{M}$ triethanolamine ( $\mathrm{pH}$ 8.2, MilliporeSigma) (59). The beads were incubated with $300 \mu \mathrm{g}$ proteins overnight at $4^{\circ} \mathrm{C}$. The following day, output was collected, and beads were washed in PBST (PBS, pH 5, Tween 0.02\%) before elution of bound proteins. Immunoprecipitation of $\mathrm{scFv}$ was performed in the same way, but the beads were coated with $2 \mu \mathrm{g}$ mouse monoclonal anti-c-Myc antibody (Santa Cruz Biotechnology).

Immunoprecipitation of ubiquitinated proteins was performed as previously described (60). Briefly, pellets of transfected cells collected from $10-\mathrm{cm}$ dishes were lysated in $200 \mu \mathrm{l}$ lysis buffer (2\% SDS, 150 $\mathrm{mM} \mathrm{NaCl}, 10 \mathrm{mM}$ Tris- $\mathrm{HCl} \mathrm{pH} 8,2 \mathrm{mM}$ sodium orthovanadate, 50 $\mathrm{mM}$ sodium fluoride supplemented with protease inhibitors). Proteins were then diluted in $800 \mu \mathrm{l}$ dilution buffer $(10 \mathrm{mM}$ Tris- $\mathrm{HCl} \mathrm{pH} 8,150$ $\mathrm{mM} \mathrm{NaCl}, 2 \mathrm{mM}$ EDTA and $1 \%$ Triton-X), resuspended for 1 hour with rotation at $4^{\circ} \mathrm{C}$, and centrifuged for 30 minutes at 20,000 g. Supernatant was collected, quantified, and used for the immunoprecipitation experiments. Magnetic beads $(30 \mu \mathrm{l})$ were coated with $10 \mu \mathrm{g}$ rabbit monoclonal anti-ubiquitin Lys48 (MilliporeSigma, rabbit monoclonal, clone Apu2, 05-1307) or Lys63-specific (MilliporeSigma, rabbit monoclonal, clone Apu3, 05-1308) antibodies for 2 hours at room temperature with rotation. Immunoprecipitation was then performed as previously described, except for the washes done in washing buffer $(10 \mathrm{mM}$ Tris-HCl, $1 \mathrm{M} \mathrm{NaCl}, 1 \mathrm{mM}$ EDTA, 1\% NP-40).

Immunoprecipitated proteins were eluted from beads by boiling for 5 minutes at $100^{\circ} \mathrm{C}$ in loading buffer supplemented with $100 \mathrm{mM}$ DTT (MilliporeSigma).

All Western blotting was performed with antibodies with Igs different from those used for immunoprecipitation to avoid cross-reactions.

Immunofluorescence and image analysis. Fixed cells or 30- $\mu \mathrm{m}$ tissue sections cut with a microtome (Leica) were washed 3 times in PBS and incubated for 1 hour with blocking solution (NGS 10\%, Triton X-100 $0.2 \%$ in PBS) and then overnight with primary antibodies diluted in blocking solution (Supplemental Table 1). After washes in PBS, the samples were incubated for 2 hours with goat-anti mouse or goat antirabbit Alexa Fluor (Invitrogen, Thermo Fisher Scientific) secondary antibodies diluted at 1:500 in blocking solution and washed again in PBS. Nuclei were visualized with a 5-minute incubation with Hoechst
33342 (Invitrogen, Thermo Fisher Scientific) diluted 1:1000 in PBS. Slides were mounted with Fluoromount-G Buffer (Southern Biotech). Images were acquired using a BX-61 Virtual Stage confocal microscope (Olympus), an Apotome microscope (Carl Zeiss), or a DM5000 $B$ wide-field microscope (Leica Microsystems).

For lumbar spinal cord sections, images were acquired focusing on the ventral horns for TDP-43 location and astrogliosis and microgliosis assessments. For TDP-43 localization analysis in brain sections, images were acquired focusing on the neurons located in the fifth layer of the primary motor cortex (Betz cells). Global microgliosis assessment was performed in all 6 layers of the primary motor cortex. All acquired brain images distinguished injected and noninjected hemispheres by making a lateral incision in the contralateral hemisphere.

For nuclear and cytoplasmic localization of TDP-43, images were analyzed with ImageJ software (NIH) as previously described (61). Briefly, for each cell analyzed, total or nuclear TDP-43 integrated density was obtained by selecting the compartment according to the neuronal (NeuN, CHAT) or nuclear (Hoechst) markers. Cytoplasmic TDP43 was obtained by substraction of the integrated nuclear density from the total. The cytoplasm to nuclear ratio was considered for analyses. For Iba1 and GFAP quantification, images were analyzed with ImageJ software. The adequate threshold was determined for all the images, and an average of them was used for the final analysis. The parameters analyzed included area fraction, number of events, and size of cells. Motoneurons, microglia and astrocytes sizes were obtained by setting the scale according to the enlargement. CHAT+ve cells larger than $250 \mu \mathrm{m}^{2}$ and Iba1+ve or GFAP+ve cells larger than 20 to $35 \mu \mathrm{m}^{2}$ were included in the analysis as previously reported (62). Neuromuscular junction (NMJ) staining was performed as previously described (29, 61) on mouse gastrocnemius muscle sections $(25-\mu \mathrm{m}$ thick) obtained by cryostat sectioning (Thermo Fisher Scientific).

Mice overexpressing the human $T D P-43^{A 315 T}$ or $T D P-43^{G 348 C}$ transgene were generated in the laboratory of JPJ (35) and maintained, after more than 20 backcrosses, on a C57/BL6 background (Charles River Laboratories). $h T D P-43^{G 348 C}$ mice ( $n=37$ females, $n=33$ males), $h T D P-43^{A 315 T}$ mice ( $n=22$ females, $n=14$ males), or nontransgenic (NTg, from the $h T D P-43^{\mathrm{G} 348 \mathrm{C}}$ line) mice ( $n=4$ females, $n=6$ males) were injected with PBS or virus at 8 months of age. Mice expressing the TLR2-luciferase transgene were generated in the laboratory of JK (34) and maintained, after more than 20 backcrosses, on a C57/BL6 Albino background (Charles River Laboratories), and nontransgenic $(\mathrm{NTg})$ mice were obtained from the same colony. Mice were injected with virus at 4 months of age ( $n=11$ TLR2-luciferase males, $n=6 \mathrm{NTg}$ males). Transgenic mice were identified by PCR of DNA tail biopsies and constantly maintained as a colony at the CERVO Brain Centre animal facility under standard conditions.

Experimental design. Mice were randomly assigned to the experimental groups, although sex was taken into consideration. Technical randomization was applied for the in vitro experiments when possible.

Data collection was performed in a blinded manner for all in vivo experiments. One researcher determined the treatment for each mouse. Virus injections were performed by experimenters blinded to the type of $\mathrm{scFv}$ produced by the virus; behavioral tests and biochemistry and immunofluorescence experiments on mice were performed only with the knowledge of their identification number and treatment group but not the type of treatment received. Data collection was not done in a blinded manner for the in vitro experiments. 
Statistics. GraphPad Prism 5.0 (GraphPad Software) was used for all statistical analyses. Data distribution was assumed to be normal but was not formally tested. Unpaired, 2-tailed $t$ tests and 1- or 2-way ANOVA followed by post hoc test for multiple comparisons correction or repeated-measures correction were performed depending on the experimental design. A $P$ value of less than 0.05 was considered statistically significant.

For quantitative analyses, the numbers of biological replicates are stated in the text. Sample size for the in vivo analyses was not determined by statistical methods but was consistent with other studies in the field (61), and biochemical and histological experiments were always performed using at least 3 individual mice for each experiment.

Data exclusion was carried out in several instances. For in vitro analyses in Figure 2B, data from 1 experiment involving control scFv+LPS were excluded because of a technical mistake. For in vivo analyses, several mice were not evaluated because of technical problems during the evaluation. Data exclusion for TDP- $43^{\mathrm{G} 348 \mathrm{C}_{-}}$ injected mice involved the following: PBS-injected mice $(n=3)$ and 8 H11-injected mice $(n=2)$ in the NOR test at 2 months; VH7Vk9injected mice $(n=1)$ in the NOR test at 4 months; VH7Vk9-injected mice $(n=3), 8 \mathrm{H} 11$-injected mice $(n=4)$, and NTg PBS-injected mice $(n=2)$ in the OFT at 4 months. For TDP- $43^{A 315 T}$-injected mice, several individual time data points were excluded: $n=4$ excluded for VH7Vk9 and $n=3$ excluded for 8 H11 among 180 data points for each group. Some values were excluded from the cytokine array analysis because they were undetectable by the assay.

Study approval. The Animal Care Ethics Committee of the Université Laval approved all in vivo experimental protocols. All experiments were performed in accordance with the Guide to the
Care and Use of Experimental Animals (Canadian Council on Animal Care, 1993, 2nd edition, revised March 2017).

\section{Author contributions}

SP and JPJ designed the study. SP performed the majority of the experiments; SST helped with behavioral tests, tissue preparation, and immunofluorescence; PC helped with immunofluorescence and image analysis, RR performed in vivo bioimaging; KVP performed UV-CLIP experiments; GS performed mouse injections; CB produced the recombinant hTDP- 43 protein; DP produced the hTDP-43 plasmids; CG produced the original pscFv9 empty plasmid, the pscFv9-8H11 scFv plasmid, and the scAAV2/9 virus; and JK provided the TLR2-luciferase mice. SP analyzed the data and wrote the manuscript. JPJ revised the manuscript. All authors read and approved the final manuscript.

\section{Acknowledgments}

SP would like to thank Kallol Dutta and Hejer Boutej for useful discussions, Louis-Charles Béland for the production of the graphical abstract, and Marie-Eve Crochetière for help during her summer training (all from CERVO Brain Research Centre). This work was funded by the Canadian Institutes of Health Research and the Packard Center for ALS Research at Johns Hopkins. JPJ holds a Canada Research Chair in Neurodegeneration.

Address correspondence to: Jean-Pierre Julien, Department of Psychiatry and Neuroscience, Faculty of Medicine, Laval University, CERVO Brain Research Centre, 2601 Chemin de la Canardière, Québec, Québec G1J 2G3, Canada. Phone: 418.663.5000 ext 4785; Email: jean-pierre.julien@fmed.ulaval.ca.
1. Morgan J. Ammar Al-Chalabi: from complex genetics to acoustic rock. Lancet Neurol. 2017;16(5):347.

2. Hardiman O, et al. Amyotrophic lateral sclerosis. Nat Rev Dis Primers. 2017;3:17071.

3. Taylor JP, Brown RH, Cleveland DW. Decoding ALS: from genes to mechanism. Nature. 2016;539(7628):197-206.

4. Buratti E. Functional Significance of TDP-43 Mutations in Disease. Adv Genet. 2015;91:1-53.

5. Burrell JR, et al. The frontotemporal dementia-motor neuron disease continuum. Lancet. 2016;388(10047):919-931.

6. Neumann M, et al. Ubiquitinated TDP-43 in frontotemporal lobar degeneration and amyotrophic lateral sclerosis. Science. 2006;314(5796):130-133.

7. Arai T, et al. TDP- 43 is a component of ubiquitin-positive tau-negative inclusions in frontotemporal lobar degeneration and amyotrophic lateral sclerosis. Biochem Biophys Res Commun. 2006;351(3):602-611.

8. Barmada SJ, Skibinski G, Korb E, Rao EJ, Wu JY, Finkbeiner S. Cytoplasmic mislocalization of TDP-43 is toxic to neurons and enhanced by a mutation associated with familial amyotrophic lateral sclerosis. J Neurosci. 2010;30(2):639-649.

9. Mackenzie IR, Neumann M. Molecular neuropathology of frontotemporal dementia: insights into disease mechanisms from postmortem studies. J Neurochem. 2016;138 Suppl 1:54-70.
10. Arai T. Significance and limitation of the pathological classification of TDP-43 proteinopathy. Neuropathology. 2014;34(6):578-588.

11. Chang CK, Chiang MH, Toh EK, Chang CF, Huang TH. Molecular mechanism of oxidation-induced TDP-43 RRM1 aggregation and loss of function. FEBS Lett. 2013;587(6):575-582.

12. Shodai A, et al. Aberrant assembly of RNA recognition motif 1 links to pathogenic conversion of TAR DNA-binding protein of $43 \mathrm{kDa}$ (TDP-43). J Biol Chem. 2013;288(21):14886-14905.

13. Swarup V, et al. Deregulation of TDP-43 in amyotrophic lateral sclerosis triggers nuclear factor $\kappa \mathrm{B}$-mediated pathogenic pathways. JExp Med. 2011;208(12):2429-2447.

14. Nardo G, et al. Amyotrophic lateral sclerosis multiprotein biomarkers in peripheral blood mononuclear cells. PLOS ONE. 2011;6(10):e25545.

15. Messer A, Joshi SN. Intrabodies as neuroprotective therapeutics. Neurotherapeutics. 2013;10(3):447-458.

16. Ahmad ZA, Yeap SK, Ali AM, Ho WY, Alitheen NB, Hamid M. scFv antibody: principles and clinical application. Clin Dev Immunol. 2012;2012:980250.

17. Esquerda-Canals G, Marti J, Rivera-Hernández G, Giménez-Llort L, Villegas S. Loss of deep cerebellar nuclei neurons in the $3 \times \mathrm{Tg}$-AD mice and protection by an anti-amyloid $\beta$ antibody fragment. MAbs. 2013;5(5):660-664.
18. Giménez-Llort L, Rivera-Hernández G, Marin-Argany M, Sánchez-Quesada JL, Villegas S. Early intervention in the $3 \times \mathrm{Tg}-\mathrm{AD}$ mice with an amyloid $\beta$-antibody fragment ameliorates first hallmarks of Alzheimer disease. MAbs. 2013;5(5):665-677.

19. Meli $G$, et al. Conformational targeting of intracellular $\mathrm{A} \beta$ oligomers demonstrates their pathological oligomerization inside the endoplasmic reticulum. Nat Commun. 2014;5:3867.

20. Sebollela A, et al. A human scFv antibody that targets neutralizes high molecular weight pathogenic amyloid-beta oligomers [published online ahead of print July 3, 2017]. J Neurochem. https://doi.org/10.1111/jnc.14118.

21. Spencer B, et al. ESCRT-mediated uptake and degradation of brain-targeted $\alpha$-synuclein single chain antibody attenuates neuronal degeneration in vivo. Mol Ther. 2014;22(10):1753-1767.

22. Spencer B, et al. $\alpha$-synuclein conformational antibodies fused to penetratin are effective in models of Lewy body disease. Ann Clin Transl Neurol. 2016;3(8):588-606.

23. Snyder-Keller A, McLear JA, Hathorn T, Messer A. Early or late-stage anti-N-terminal Huntingtin intrabody gene therapy reduces pathological features in B6.HDR6/1 mice. J Neuropathol Exp Neurol. 2010;69(10):1078-1085.

24. Butler DC, Messer A. Bifunctional anti-huntingtin proteasome-directed intrabodies mediate 
efficient degradation of mutant huntingtin exon 1 protein fragments. PLoS One. 2011;6(12):e29199.

25. Ghadge GD, Pavlovic JD, Koduvayur SP, Kay BK, Roos RP. Single chain variable fragment antibodies block aggregation and toxicity induced by familial ALS-linked mutant forms of SOD1. Neurobiol Dis. 2013;56:74-78.

26. Patel P, et al. Adeno-associated virus-mediated delivery of a recombinant single-chain antibody against misfolded superoxide dismutase for treatment of amyotrophic lateral sclerosis. Mol Ther. 2014;22(3):498-510.

27. Tamaki Y, et al. Elimination of TDP-43 inclusions linked to amyotrophic lateral sclerosis by a misfolding-specific intrabody with dual proteolytic signals. Sci Rep. 2018;8(1):6030.

28. Ayala YM, et al. TDP- 43 regulates its mRNA levels through a negative feedback loop. $Е М B O J$. 2011;30(2):277-288.

29. Dutta K, Patel P, Rahimian R, Phaneuf D, Julien JP. Withania somnifera reverses transactive response DNA binding protein 43 proteinopathy in a mouse model of amyotrophic lateral sclerosis/frontotemporal lobar degeneration. Neurotherapeutics. 2017;14(2):447-462.

30. Frakes AE, et al. Microglia induce motor neuron death via the classical NF- $\kappa$ B pathway in amyotrophic lateral sclerosis. Neuron. 2014;81(5):1009-1023.

31. Urushitani M, Sato T, Bamba H, Hisa Y, Tooyama I. Synergistic effect between proteasome and autophagosome in the clearance of polyubiquitinated TDP-43. JNeurosci Res. 2010;88(4):784-797.

32. Scotter EL, et al. Differential roles of the ubiquitin proteasome system and autophagy in the clearance of soluble and aggregated TDP-43 species. J Cell Sci. 2014;127(Pt 6):1263-1278.

33. Iguchi $Y$, et al. Oxidative stress induced by glutathione depletion reproduces pathological modifications of TDP- 43 linked to TDP-43 proteinopathies. Neurobiol Dis. 2012;45(3):862-870.

34. Lalancette-Hébert M, Phaneuf D, Soucy G, Weng YC, Kriz J. Live imaging of Toll-like receptor 2 response in cerebral ischaemia reveals a role of olfactory bulb microglia as modulators of inflammation. Brain. 2009;132(Pt 4):940-954.

35. Swarup V, et al. Pathological hallmarks of amyotrophic lateral sclerosis/frontotemporal lobar degeneration in transgenic mice produced with TDP-43 genomic fragments. Brain. 2011;134(Pt 9):2610-2626.

36. Fiesel FC, Weber SS, Supper J, Zell A, Kahle PJ.
TDP-43 regulates global translational yield by splicing of exon junction complex component SKAR. Nucleic Acids Res. 2012;40(6):2668-2682.

37. Sephton CF, et al. Identification of neuronal RNA targets of TDP-43-containing ribonucleoprotein complexes. J Biol Chem. 2011;286(2):1204-1215.

38. Strong MJ, et al. TDP 43 is a human low molecular weight neurofilament (hNFL) mRNA-binding protein. Mol Cell Neurosci. 2007;35(2):320-327.

39. Manoutcharian K, Perez-Garmendia R, Gevorkian G. Recombinant antibody fragments for neurodegenerative diseases. Curr Neuropharmacol. 2017;15(5):779-788.

40. Ghadge GD, Kay BK, Drigotas C, Roos RP. Single chain variable fragment antibodies directed against SOD1 ameliorate disease in mutant SOD1 transgenic mice. Neurobiol Dis. 2019;121:131-137.

41. Dong QX, Zhu J, Liu SY, Yu XL, Liu RT. An oligomer-specific antibody improved motor function and attenuated neuropathology in the SOD1-G93A transgenic mouse model of ALS. Int Immunopharmacol. 2018;65:413-421.

42. Walker AK, et al. Functional recovery in new mouse models of ALS/FTLD after clearance of pathological cytoplasmic TDP-43. Acta Neuropathol.2015;130(5):643-660.

43. Thomas M, Alegre-Abarrategui J, Wade-Martins R. RNA dysfunction and aggrephagy at the centre of an amyotrophic lateral sclerosis/frontotemporal dementia disease continuum. Brain. 2013;136(Pt 5):1345-1360.

44. Budini M, Baralle FE, Buratti E. Targeting TDP43 in neurodegenerative diseases. Expert Opin Ther Targets. 2014;18(6):617-632.

45. Scotter EL, Chen HJ, Shaw CE. TDP-43 proteinopathy and ALS: insights into disease mechanisms and therapeutic targets. Neurotherapeutics. 2015;12(2):352-363.

46. Wang IF, et al. Autophagy activators rescue and alleviate pathogenesis of a mouse model with proteinopathies of the TAR DNA-binding protein 43. Proc Natl Acad Sci U S A. 2012;109(37):15024-15029.

47. Barmada SJ, et al. Autophagy induction enhances TDP43 turnover and survival in neuronal ALS models. Nat Chem Biol. 2014;10(8):677-685.

48. Guo J, Yang X, Gao L, Zang D. Evaluating the levels of CSF and serum factors in ALS. Brain Behav. 2017;7(3):e00637.

49. Rentzos M, et al. Interleukin-15 and interleukin-12 are elevated in serum and cerebrospinal fluid of patients with amyotrophic lateral sclerosis. Eur Neurol. 2010;63(5):285-290.

50. Rentzos M, et al. Interleukin-17 and interleukin-23 are elevated in serum and cerebrospinal fluid of patients with ALS: a reflection of Th17 cells activation? Acta Neurol Scand. 2010;122(6):425-429.

51. Cereda C, et al. TNF and sTNFR1/2 plasma levels in ALS patients. J Neuroimmunol. 2008;194(1-2):123-131.

52. Rentzos M, et al. RANTES levels are elevated in serum and cerebrospinal fluid in patients with amyotrophic lateral sclerosis. Amyotroph Lateral Scler. 2007;8(5):283-287.

53. Yang X, Gao L, Wu X, Zhang Y, Zang D. Increased levels of MIP-1 $\alpha$ in CSF and serum of ALS. Acta Neurol Scand. 2016;134(2):94-100.

54. McCarty DM, Monahan PE, Samulski RJ. Self-complementary recombinant adeno-associated virus (scAAV) vectors promote efficient transduction independently of DNA synthesis. Gene Ther. 2001;8(16):1248-1254.

55. Rabinowitz JE, et al. Cross-packaging of a single adeno-associated virus (AAV) type 2 vector genome into multiple AAV serotypes enables transduction with broad specificity. JVirol. 2002;76(2):791-801.

56. Lucchetti J, et al. A mouse model of familial ALS has increased CNS levels of endogenous ubiquinol9/10 and does not benefit from exogenous administration of ubiquinol10. PLoS One. 2013;8(7):e69540.

57. Iguchi $Y$, et al. Exosome secretion is a key pathway for clearance of pathological TDP-43. Brain. 2016;139(Pt 12):3187-3201.

58. Gainetdinov RR, Wetsel WC, Jones SR, Levin ED, Jaber M, Caron MG. Role of serotonin in the paradoxical calming effect of psychostimulants on hyperactivity. Science. 1999;283(5400):397-401.

59. Lauranzano E, et al. Peptidylprolyl isomerase A governs TARDBP function and assembly in heterogeneous nuclear ribonucleoprotein complexes. Brain. 2015;138(Pt 4):974-991.

60. Choo YS, Zhang Z. Detection of protein ubiquitination. J Vis Exp. 2009;(30):1293.

61. Pozzi S, Thammisetty SS, Julien JP. Chronic administration of pimozide fails to attenuate motor and pathological deficits in two mouse models of amyotrophic lateral sclerosis. Neurotherapeutics. 2018;15(3):715-727.

62. Pasetto L, et al. Targeting extracellular cyclophilin A reduces neuroinflammation and extends survival in a mouse model of amyotrophic lateral sclerosis. J Neurosci. 2017;37(6):1413-1427. 